\title{
Tittle Exploring the Mechanism of Resveratrol on Traumatic Brain Injury by Network Pharmacology and Molecular Docking
}

\section{Feifei Liu}

Hangzhou Hospital of Traditional Chinese Medicine

Lijun Han

Hangzhou Hospital of Traditional Chinese Medicine

\section{Yujian Li}

Hangzhou Hospital of Traditional Chinese Medicine

Guangming Bao ( $\nabla$ yimeng-yimeng@hotmail.com )

Hangzhou Hospital of Traditional Chinese Medicine https://orcid.org/0000-0003-4887-6696

\section{Research}

Keywords: Resveratrol, Traumatic brain injury, Network pharmacology, Molecular docking, Bioinformatics

Posted Date: December 1st, 2020

DOI: https://doi.org/10.21203/rs.3.rs-113815/v1

License: (c) (i) This work is licensed under a Creative Commons Attribution 4.0 International License. Read Full License 


\section{Abstract}

[Background] Resveratrol is a polyphenol present abundantly in lots of traditional Chinese medicines, which has been shown to have beneficial effects on neurological diseases. However, the molecular mechanisms of resveratrol on traumatic brain injury have not been systematically studied yet. In this study, we elucidated the pharmacological mechanisms of resveratrol in treating traumatic brain injury by using a network pharmacology method.

[Methods] The pharmacokinetics properties of resveratrol were obtained from the traditional Chinese medicine systems pharmacology database and analysis platform (TCMSP). The putative targets of resveratrol were obtained from TCMSP, BATMAN-TCM, SuperPred, PharmMapper, SwissTargetPrediction, DrugBank, and a literature search. The targets related to traumatic brain injury were obtained from TTD, DrugBank, CTD, GeneCards, OMIM, MalaCards, and a literature search. The STRING database and the Cytoscape 3.8.0 software were used to build the protein-protein interaction (PPI) network. The Metascape database was used to obtain the gene ontology (GO) function and Kyoto Encyclopedia of Genes and Genomes (KEGG) pathway enrichment information. Finally, The AutoDockTools 1.5.6 and PyMOL 2.4.0 software were employed for molecular docking analyses, and Discovery Studio 2020 was used for interaction analyses.

[Results] A total of 165 overlapping targets involved in resveratrol intervention in traumatic brain injury were determined. The $\mathrm{GO}$ function analysis indicated that the targets are including the positive regulation of transferase activity, the positive regulation of cell migration, reactive oxygen species metabolism, the wound response, and so on. The KEGG pathway analysis identified the following enriched pathways: the AGE-RAGE signalling pathway, the FoxO signalling pathway, insulin resistance, complement and coagulation cascades, the HIF-1 signalling pathway, and so on. According to the PPI network analysis, INS, IGF1, TNF, TP53, ALB, IL6, SRC, STAT3, VEGFA, and MMP9 were identified as hub target genes, in which IL6, MMP9, INS, and SRC showed a good binding affinity with resveratrol in molecular docking.

[Conclusions] Resveratrol may target multiple genes and multiple pathways to reduce brain damage after traumatic brain injury.

\section{Background}

Traumatic brain injury (TBI) refers to damage to the brain caused by a violent force applied directly or indirectly to the head ${ }^{[1]}$. Because TBI often leads to complications such as infection, limb motor dysfunction, brain nerve dysfunction, and systemic organ dysfunction, the mortality rate of TBI in China is as high as $7 \%{ }^{[2]}$. The pathological mechanism of TBI is complicated, and there is no effective treatment for TBI in the clinic. Therefore, the development of high-potency therapeutic drugs is a new focus of TBI research. Resveratrol is a nonflavonoid polyphenol compound with multiple pharmacological activities, such as antitumour, antioxidant, anti-inflammatory, antiaging, immune regulatory, and neuroprotective activities. In the nervous system, resveratrol inhibits the NF-kB pathway, 
activates the mammalian sir2-related protein 1 (Sirt1) pathway, regulates autophagy signalling, inhibits the release of inflammatory factors, and has a neuroprotective effect ${ }^{[3]}$. The concept of network pharmacology was first proposed by Hopkins in 2007. Network pharmacology integrates systems biology, pharmacology, and information science to construct a "drug-target-disease" interaction network that can then be used to systematically and integratively study the links among drugs, targets, and disease phenotypes. Network pharmacology provides a new method for the research and development of new drugs. Therefore, this study uses network pharmacology methods to screen the possible target genes of resveratrol in the treatment of TBI and uses molecular docking technology to verify the targets, thereby providing a reference basis for the treatment of TBI and the clinical application of resveratrol.

\section{Methods}

\subsection{The prediction of putative targets of resveratrol}

In this research, pharmacokinetics parameters of resveratrol were retrieved through the traditional Chinese medicine systems pharmacology database and analysis platform (TCMSP, https://tcmspw.com/tcmsp.php) ${ }^{[4]}$. The TCMSP, BATMAN-TCM (http://bionet.ncpsb.org/batman-tcm/) [5], DrugBank (https://go.drugbank.com/) ${ }^{[6]}$, SuperPred (http://prediction.charite.de/) ${ }^{[7]}$, PharmMapper (http://www.lilab-ecust.cn/pharmmapper/) ${ }^{[8]}$, and SwissTargetPrediction (http://www.swisstargetprediction.ch/) ${ }^{[9]}$ databases were searched to obtain the resveratrol targets, and the CNKI (https://www.cnki.net/), WAN FANG (http://www.wanfangdata.com.cn/index.html), CQVIP (http://www.cqvip.com/), and PubMed (https://www.ncbi.nlm.nih.gov/) databases were manually searched to identify additional resveratrol targets.

\subsection{Collection of TBI-related targets}

The TTD (http://db.idrblab.net/ttd/) ${ }^{[10]}$, DrugBank, CTD (http://ctdbase.org/) ${ }^{[11]}$, GeneCards (https://www.genecards.org/) ${ }^{[12]}$, OMIM (https://omim.org/) ${ }^{[13]}$ and MalaCards (https://www.malacards.org// ${ }^{[14]}$ databases were searched using the following keywords: "Traumatic Brain Injury", "Brain Injury, Traumatic", "Trauma, Brain", "Intracranial Trauma", "Craniocerebral Trauma", "Brain Trauma" or "Encephalopathy, Traumatic" to collect targets related to TBI. The literature related to the targets influenced by TBI is reviewed in the supplement.

\subsection{Construction of the drug-target-disease network}

The TBI-related and resveratrol targets were uploaded to the UniProt database (https://www.UniProt.org/) ${ }^{[15]}$ to convert them into SYMBOL gene names, and just "Homo sapiens" genes were selected. The Bioinformatics website (http://www.bioinformatics.com.cn/) was used to construct a Venn diagram, and the overlapping genes were the target genes of resveratrol in the treatment of TBI. The overlapping genes were imported into the Cytoscape 3.8.0 software (https://www.cytoscape.org/) to construct a drug-targetdisease network map. 


\subsection{Construction of the Protein-Protein-Interaction (PPI) network}

All the targets of resveratrol in the treatment of TBI were imported into the STRING (https://string-db.org/)

[16] database to construct a PPI network. The protein type was set to "Homo sapiens", the lowest interaction threshold was set to "medium confidence $(0.40)$ ", the unlined targets were hidden, and the other parameters were kept at their default values. The PPI network was imported into the Cytoscape 3.8.0 software, and the "Network Analysis" function was employed to calculate important network topology parameters such as the degree, combined score, and betweenness of the protein nodes. The CytoHubba plug-in, which is based on the maximum clique centrality (MCC) calculation method, was used to screen the top 10 nodes in the PPI network, which were defined as the hub genes. The MCODE plug-in was used to perform cluster analysis of the PPI network and to select the functional modules based on the number of nodes and the k-core value.

2.5 GO function and KEGG pathway enrichment analyses

GO function enrichment and KEGG pathway analysis were used to understand the biological functions of the gene products. The overlapping targets and functional modules were imported into the Metascape database (https://metascape.org/gp/index.html) ${ }^{[17]}$, the species was set to "Homo sapiens", and the following aspects were selected for the custom analysis: GO Biological Processes (BP), Molecular Functions (MF), Cellular Components (CC), and KEGG Pathway. The threshold was set to $P<0.05$, and the GO items and KEGG pathways were screened according to the P-values. Cytoscape 3.8.0 was used to construct a drug-target-KEGG pathway network diagram, and the Bioinformatics and ImageGP websites (http://www.ehbio.com/ImageGP/index.php/Home/Index/index.html) were used to visualize the GO function enrichment and KEGG pathway analysis results.

\subsection{Molecular docking of the hub targets and resveratrol}

The hub targets and resveratrol were selected for the molecular docking analysis. The 3D structure of resveratrol was downloaded from the ZINC database (http://zinc.docking.org/) ${ }^{[18]}$ and saved in the Mol2 format as an active small molecule in molecular docking. The protein structures of the hub targets were downloaded from the RCSB PDB database (http://www.rcsb.org/) ${ }^{[19]}$. The PyMOL 2.4.0 software was used to perform operations such as dewatering, removing the original ligand, and hydrogenating the protein, and the file was then saved in the PDB format. The AutoDockTools 1.5.6 software was used for semi-flexible docking; the docking results for the highest-scoring conformation were visualized with PyMOL 2.4.0, and the interaction analysis was performed with Discovery Studio 2020.

\section{Results}

\subsection{Pharmacokinetics parameters of resveratrol}

Detailed information on the oral bioavailability (OB), drug-likeness (DL) properties, blood-brain barrier (BBB) permeability, and other attributes of resveratrol was obtained by querying the TCMSP database. 
The results are shown in Table 1. Although resveratrol has low OB and DL values, it has been reported in the literature that resveratrol may have therapeutic effects in neurodegenerative diseases [20]; additionally, the US FDA has approved resveratrol as an anticancer and antioxidant agent for use in clinical treatment, showing that resveratrol has medicinal properties.

Table 1 Pharmacological and molecular properties of resveratrol

\begin{tabular}{|llllll|}
\hline Molecule ID & MW & OB (\%) & Caco-2 & BBB & DL \\
\hline MOL012744 & 228.26 & 19.07 & 0.80 & -0.01 & 0.11 \\
\hline
\end{tabular}

\subsection{Screening of resveratrol- and TBI-related targets}

A total of 577 resveratrol targets were obtained by searching the TCMSP, BATMAN-TCM, DrugBank, SuperPred, PharmMapper, and SwissTargetPrediction databases and conducting a literature search. A total of 9231 TBI-related targets were retrieved from the GeneCards database, and a total of 845 targets with a relevance score greater than 20 were selected. The targets were supplemented with 255 additional targets by searching the TTD, DrugBank, CTD, OMIM, and MalaCards databases. After the targets were reviewed, $1100 \mathrm{TBI}$-related targets were ultimately selected. The resveratrol and TBI-related targets were intersected, and a total of 165 targets of resveratrol in the treatment of TBI were obtained (Figure 1).

Fig 1 The overlapping targets of resveratrol and TBI

\subsection{Drug-target-disease network construction}

The resveratrol targets, the TBI-related targets, and the 165 resveratrol targets in TBI were introduced into Cytoscape 3.8.0, and a drug-target-disease network diagram was constructed (Figure 2) that involved 1,514 nodes and 1,677 edges. Among the nodes, the orange node represents the resveratrol target category, the yellow node represents the TBI-related target category, the green nodes represents the individual resveratrol targets, the blue nodes represent the individual TBI-related targets, the brown nodes represents the targets of resveratrol in the treatment of $\mathrm{TBI}$, and the edges represent the interrelationships among the drug, targets, and disease. The figure shows that resveratrol may affect TBI by acting on multiple targets.

Fig 2 Drug-target-disease network of resveratrol in TBI treatment

3.4. PPI network construction and module analysis of resveratrol targets in TBI.

The STRING database and Cytoscape 3.8.0 software were used to construct the PPI network for the 165 overlapping targets, and a network with 165 nodes and 3672 edges was obtained (Figure 3 ). The "Network Analyzer" function was used to analyse the network and evaluate the importance of nodes with their degrees and combined scores. The nodes in the figure represent the targets, and the edges represent the associations between targets. The size of each node is proportional to its degree value. The larger the 
degree value, the larger the node, and the darker the colour. The thickness of the edge is directly proportional to the combined score. The larger the combined score, which indicates a closer connection between related targets, the thicker the edge and the darker the colour. It can be seen that there are interactions among these 165 targets, which indicates that these targets are related to each other and affect TBI through mutual coordination.

Fig 3 The PPI network of overlapping targets of resveratrol and TBI

The MCODE plug-in was used to analyse the functional modules of the PPI network, and 5 functional modules with nodes $\geq 4$ and k-core $\geq 3$ (MCODE 1-5) were identified (Figure 4). The MCODE 1 module contains 54 nodes and 1265 edges. MCODE 2 contains 26 nodes and 82 edges. MCODE 3 contains 8 nodes and 20 edges. MCODE 4 contains 6 nodes and 9 edges. MCODE 5 contains 5 nodes and 6 edges.

Fig 4 Module analysis of overlapping targets

To further analyse the functions of the above five modules, the targets of the modules were imported into the Metascape database for GO enrichment and KEGG pathway analysis and sorted according to $\log 10(p)$, and the first 20 entries were selected for inclusion in the diagram (Figure 5).

Fig 5 GO functional and KEGG pathway enrichment analysis of MCODE 1-5

The results of the functional module enrichment analysis show that the biological processes associated with MCODE 1 are mainly related to the positive regulation of cell migration, epithelial cell proliferation, response to oxidative stress, cytokine-mediated signalling, blood vessel development, and so on. The molecular functions associated with MCODE 1 are mainly involved in kinase, cytokine receptor, transcription factor, and phosphatase binding; protein kinase activity; and so on. The main cell components associated with MCODE 1 include membrane rafts, vesicle lumens, focal adhesions, RNA polymerase II transcription factor complexes, the basal part of the cell, and so on. The signalling pathways associated with MCODE 1 include pathways in cancer, the AGE-RAGE signalling pathway in diabetic complications, hepatitis B, endocrine resistance, EGFR tyrosine kinase inhibitor resistance, fluid shear stress and atherosclerosis, the FoxO signalling pathway, the HIF-1 signalling pathway, the JAKSTAT signalling pathway, insulin resistance, and so on. The biological processes associated with MCODE 2 are mainly related to the cellular response to hormone stimuli, phosphatidylinositol 3-kinase signalling, reactive oxygen species metabolism, the regulation of hormone secretion, the positive regulation of kinase activity, and so on. The molecular functions associated with MCODE 2 are mainly involved in insulin receptor substrate binding, glycosaminoglycan binding, $\mathrm{SH} 2$ domain binding, protein domainspecific binding, antioxidant activity, and so on. The main cell components associated with MCODE 2 include cell-cell junctions, transferase complexes, transferrin phosphorus-containing groups, membrane rafts, lytic vacuoles, receptor complexes, and so on. The signalling pathways associated with MCODE 2 include pathways in cancer, insulin resistance, adipocytokine signalling, peroxisomes, and neuroactive ligand-receptor interactions. The biological processes associated with MCODE 3 involve dopamine metabolism, organic hydroxy compound metabolism, monoamine transport, drug catabolism, and 
cofactor biosynthesis; the molecular functions associated with MCODE 3 include $\mathrm{G}$ protein-coupled amine receptor activity and oxidoreductase activity; the MCODE 3-associated cell components include glutamatergic synapses and dendrites; and the signalling pathways associated with MCODE 3 include serotonergic and dopaminergic synapses and neuroactive ligand-receptor interactions. The biological processes associated with MCODE 4 are related to behaviour, second-messenger-mediated signalling, the alcohol response, and the activation of protein kinase activity; the molecular functions associated with MCODE 4 are related to peptide binding; the MCODE-4-associated cell components include axons, membrane rafts, neuron cell bodies, and presynapses; and the signalling pathways associated with MCODE 4 are related to neuroactive ligand-receptor interaction. The biological processes associated with MCODE 5 are related to cell-matrix adhesion, axon guidance, the positive regulation of protein kinase activity, cell division, lymphocyte activation, and so on; the molecular functions associated with MCODE 5 are related to protein kinase activity and kinase binding; the MCODE 5-associated cell components include serine/threonine protein kinase complexes, the leading edge of the cell, and dendrites; and the signalling pathways associated with MCODE 5 are involved in viral carcinogenesis and cancer.

\subsection{GO function and KEGG pathway enrichment analyses of targets for resveratrol against TBI}

The 165 overlapping targets were imported into the Metascape database for GO functional and KEGG pathway enrichment analysis and sorted according to $-\log 10(\mathrm{p})$, and the top 20 hits for each item were kept; the Bioinformatics platform was used to draw the GO bar graphs (Figure 6) and KEGG pathway bubble graphs (Figure 7). The results show that the targets are enriched in 303 biological processes, including the positive regulation of transferase activity, responses to toxic and inorganic substances, the positive regulation of cell migration, reactive oxygen species metabolism, the wound response, epithelial cell proliferation and so on. The targets are enriched in 117 molecular functions, including kinase binding, protein kinase activity, protein phosphatase binding, cytokine receptor binding, protein homodimerization, protease binding, transcription factor binding, and so on. The targets are enriched in 97 cell components, including membrane rafts, vesicle lumens, postsynapses, dendrites, receptor complexes, focal adhesions, endoplasmic reticulum lumens, cell-cell junctions, and so on. The targets are enriched in 148 signalling pathways, including pathways in cancer; the AGE-RAGE signalling pathway in diabetic complications; microRNAs in cancer, the FoxO signalling pathway, transcriptional misregulation in cancer, measles, and so on. The results show that resveratrol can play a role in the treatment of TBI by participating in the regulation of a variety of biological processes and multiple pathways.

Fig $6 \mathrm{GO}$ functional enrichment analysis of overlapping targets

Fig 7 KEGG pathway enrichment analysis of overlapping targets

\subsection{Construction of the drug-target-KEGG pathway network}

The drug-target-KEGG pathway network was constructed using the Cytoscape 3.8 .0 software, and the network contained 186 nodes and 734 edges. In Figure 8, the orange node represents the drug, the yellow nodes represent the targets, the green nodes represent the pathway, and the lines represent the 
relationships between the nodes. The results show that the targets of resveratrol are distributed among different pathways and participate in the pathological process of TBI through multiple targets acting on multiple pathways.

Fig 8 Drug-target-KEGG pathway network

3.7. Screening of the hub genes involved in the effect of resveratrol on TBI

The hub genes, as important nodes, play an important role in the PPI network. The "CytoHubba" plug-in was used to analyse the topology of the nodes in the PPI network, and the top 10 nodes in the network were selected based on the MCC calculation method. The results showed that insulin (INS), human insulin-like growth factor 1 (IGF1), tumor necrosis factor (TNF), tumor suppressor P53 (TP53), plasma albumin (ALB), interleukin 6 (IL6), SRC, signal transducer and activator of transcription 3 (STAT3), vascular endothelial growth factor A (VEGFA) and matrix metalloproteinase 9 (MMP9) may be the hub genes of resveratrol in TBI (Figure 9).

Fig 9 PPI network of the top 10 hub genes

\subsection{Molecular docking between resveratrol and the hub genes}

The AutoDockTools 1.5.6 software was used to verify the molecular docking between resveratrol and the 10 selected hub target proteins. If the binding free energy required for the interaction between the ligand and the receptor is less than $0 \mathrm{kcal}^{\circ} \mathrm{mol}^{-1}$, it indicates that the two can bind spontaneously. If the binding free energy is less than $-5 \mathrm{kcal} \cdot \mathrm{mol}^{-1}$, it indicates that the two have good binding affinity. The smaller the binding free energy, the more stable the conformation formed by the ligand and the receptor. The results of the molecular docking analysis showed that resveratrol and the 10 hub target proteins can all be successfully docked (Table 2). Among them, the binding free energy of resveratrol with the IL6, MMP9, INS, and SRC proteins is less than $-5 \mathrm{kcal}^{\circ} \mathrm{mol}^{-1}$, showing a good binding affinity between them. The optimal binding configurations of resveratrol with the IL6, MMP9, INS, and SRC proteins were selected for further interaction analysis. The results showed that resveratrol can form hydrogen bonds with the amino acid residues LEU65 and GLU173 and form hydrophobic interactions with the residues PRO66, LEU166, and MET68 in IL6 (Figure 10 a-b); resveratrol can form hydrogen bonds with the key residue LEU418 and hydrophobic interactions with ARG424 and GLU416 in MMP9 (Figure $10 \mathrm{c}$-d); resveratrol forms 4 hydrogen bonds with INS, including CYS7, CYS6, CYS11 and SER9, and hydrophobic interactions with the LEU11, ALA14, LEU16, and LEU13 residues (Figure 10 e-f); resveratrol and SRC form hydrogen bonds between the GLU342 and MET344 residues and hydrophobic interactions between LEU396, VAL284, ALA296 and LEU276 (Figure $10 \mathrm{~g}$-h).

Table 2 Information on molecular docking of resveratrol and 10 hub target proteins 


\begin{tabular}{|lll|}
\hline Ligand & Receptor & Affinity $\left(\mathrm{kcal} \cdot \mathrm{mol}^{-1}\right)$ \\
\hline Resveratrol & IL6 & -5.43 \\
\cline { 2 - 3 } & INS & -5.28 \\
\cline { 2 - 3 } & MMP9 & -5.08 \\
\hline SRC & -5.01 \\
\hline TNF & -4.63 \\
\hline ALB & -4.25 \\
\hline IGF1 & -3.94 \\
\hline STAT3 & -3.76 \\
\hline TP53 & -3.55 \\
VEGFA & -3.42 \\
\hline
\end{tabular}

Fig 10 The 2D and 3D molecular docking patterns of resveratrol with IL6 (a, b), MMP9 (c, d), INS (e, f), and SRC $(g, h)$

\section{Discussion}

Currently, TBI is the leading cause of death and disability in developing countries. With the in-depth research being conducted on the pathophysiological mechanism of $\mathrm{TBI}$, erythropoietin, prostacyclin, progesterone, and the ketogenic diet have been investigated in clinical research as treatments for TBI, but few drugs have passed phase III clinical trials. Resveratrol has strong antioxidant and anti-inflammatory effects and can improve cerebral blood flow and cognitive function. Experimental studies of the TBI model have shown that resveratrol can reduce the expression of TNF- $\alpha$ and IL- $1 \beta$ in the brain of TBI model rats, inhibit neuronal autophagy, reduce neuronal apoptosis, reduce brain oedema, and promote VEGF expression, which supports cell repair and the recovery of nerve function ${ }^{[21]}$. Therefore, it is of great importance to use the methods of network pharmacology to deeply explore the target genes of resveratrol and their mechanisms of action in the treatment of TBI.

This study found through database searches that there are 165 targets of resveratrol in the treatment of $\mathrm{TBI}$, and there is an interactive relationship between the targets, indicating that resveratrol has the characteristics of multitarget synergy in the treatment of TBI. The functional module and overlapping targets analyses yielded basically the same results. These targets are mainly involved in kinase binding, protein kinase activity, protein phosphatase binding, cytokine receptor binding, transcription factor binding, G protein-coupled receptor binding, and other molecular reactions in the membrane raft, vesicle lumen, postsynapse, dendrite, focal adhesion, or cell-cell junction; these targets are involved in the positive regulation of transferase activity and cell migration, reactive oxygen species metabolism, the 
wound response, the oxidative stress response, cytokine-mediated signalling and other biological processes. Resveratrol may act on various cellular components in the body and participate in various biological processes in different reaction modes. The pathways closely related to the treatment of TBI with resveratrol mainly include the AGE-RAGE signalling pathway in diabetic complications, the FoxO signalling pathway, insulin resistance, the HIF-1 signalling pathway, complement and coagulation cascades, the calcium ion signalling pathway, and the JAK-STAT signalling pathway. In addition, the targets of resveratrol may also be involved in cancer-related pathways, Alzheimer's disease, serotonergic synapses, dopaminergic synapses, neuroactive ligand-receptor interactions, fluid shear stress and atherosclerosis, the prolactin signalling pathway, the cell cycle, ovarian steroidogenesis, and other pathways. By constructing a drug-target-KEGG pathway network, it was further confirmed that resveratrol affects multiple targets and multiple pathways in the treatment of TBI.

The AGE-RAGE signalling pathway is closely related to inflammation, oxidative stress, and cell apoptosis. RAGE is present in neuronal, endothelial, glial, and immune cells, among others. After specifically binding to AGE, RAGE mediates intracellular oxidative stress, the activation of the NF-KB pathway, and the increased expression of cytokines such as TNF- $\alpha$ and IL1. This leads to neurovascular damage and nerve cell apoptosis ${ }^{[22]}$. The FoxO protein is a transcription factor that regulates cellular protein expression. FoxO plays a role in cell proliferation, autophagy, and oxidative stress by regulating the expression of downstream genes. After TBI, the expression of FoxO in brain tissue increases, and the FoxO signalling pathway regulates nerve cell autophagy to reduce nerve damage ${ }^{[23]}$. Studies have found that the FoxO signalling pathway regulated by resveratrol can reduce the release of TNF- $\alpha$ and IL-1 $\beta$ and promote the release of the anti-apoptotic factor $\mathrm{Bcl}-2$, thereby reducing cerebral ischaemia-reperfusion injury [24] Insulin resistance refers to the decrease in the body's sensitivity and responsiveness to insulin. The mitogen-activated protein kinase (MAPK) and phosphatidylinositol-3 kinase (PI3K) pathways, which are mediated by the binding of insulin to the receptors, are involved in the post-TBI stress response. Insulin resistance can aggravate lactic acidosis and calcium ion overload after TBI and then aggravate brain oedema and nerve damage ${ }^{[25]}$. The complement system is an important part of the body's immune response that has immune surveillance and immune defence effects in the brain. The destruction of the blood-brain barrier, the formation of antigen-antibody complexes, and the damage of vascular endothelial cells after TBI lead to the activation of the complement system in the brain. The activated complement system aggravates brain oedema and neuronal cell death in a variety of ways ${ }^{[26]}$. The coagulation cascade involves processes such as coagulation, anticoagulation, and fibrinolysis. After TBI, the bloodbrain barrier is broken, and the coagulation factors in the brain tissue enter the blood circulation system and activate the coagulation cascade system. Thrombin is a key component of the coagulation cascade. In addition to participating in the coagulation process after TBI, thrombin also interacts with thrombin receptors to activate a series of intracellular signal transduction pathways to induce nerve regeneration, promote angiogenesis, and mediate neurotoxicity and inflammatory reactions ${ }^{\text {[27] }}$. Blood coagulation and fibrinolytic dysfunction are closely related to progressive cerebral haemorrhage and poor prognosis after $\mathrm{TBI}^{[28]}$. In the brain, the complement system interacts with the coagulation cascade; the complement system can activate the coagulation cascade to produce thrombin, and thrombin and serotonin in the 
coagulation cascade can also activate the complement cascade. Therefore, the complement and coagulation cascade pathways interact to co-ordinately regulate the coagulation-fibrinolysis balance and immune response after TBI ${ }^{[29]}$. HIF-1 is a transcription factor that regulates the cellular response to hypoxia. HIF-1 plays important biological roles in angiogenesis, cell metabolism, inflammation, and autophagy by regulating the transcription of its corresponding hypoxia susceptibility genes. HIF-1 is widely involved in many signalling pathways, including the PI3K, MAPK, and NF-KB pathways, among others. After TBI, HIF-1 has a two-way regulatory effect. HIF-1 can not only promote red blood cell proliferation and angiogenesis by inducing VEGF expression and increase nerve cell tolerance ${ }^{\left[{ }^{[3]}\right.}$ but also mediate cell apoptosis and inflammation, leading to nerve cell death ${ }^{[31]}$. Studies have found that resveratrol can affect the permeability of the blood-brain barrier by regulating the expression of HIF-1, further verifying the reliability of the predictions made in this study ${ }^{[32]}$. The calcium ion is an important messenger in nerve cells, and it participates in activities such as maintaining nerve excitability, synthesizing neurotransmitters, and signal transduction. During TBI, the intracellular calcium ion concentration increases sharply, causing calcium ion overload, which in turn leads to nerve cell oedema and death. Calcium ion overload also exists in microvascular endothelial cells, destroys the integrity of the blood-brain barrier, and exacerbates the occurrence of vasogenic cerebral oedema after TBI. It is currently believed that calcium ion overload plays an important role in the occurrence of secondary brain

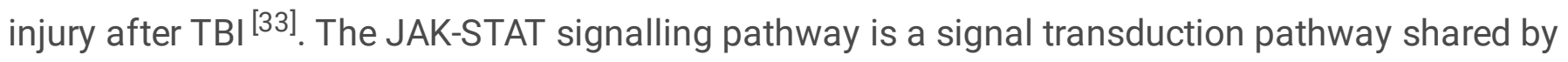
multiple cytokines that exist in almost all cytokine signal transduction processes. The JAK-STAT signalling pathway has the dual functions of signal transduction and transcriptional regulation and plays an important role in regulating cell proliferation, immune regulation, inflammation, tumours, and other pathophysiological processes. The JAK-STAT signalling pathway is activated in brain tissue after TBI and aggravates the destruction of the blood-brain barrier and nerve damage ${ }^{[34]}$.

The hub genes may be the key targets of resveratrol in the treatment of TBI. In this study, a total of 10 hub genes were identified, namely, INS, IGF1, TNF, TP53, ALB, IL6, SRC, STAT3, VEGFA, and MMP9. There is a clear interaction between the key targets, most of which are enriched in the important signalling pathways of resveratrol in the treatment of TBI. The key target protein products are involved in various physiological and biochemical processes, such as the inflammatory response, immune regulation, apoptosis, signal transduction, nerve regeneration, and wound repair. The molecular docking results showed that resveratrol can successfully bind to the 10 key target proteins. Among them, resveratrol has good binding affinity with the IL6, MMP9, INS, and SRC proteins, suggesting that resveratrol may exert a pharmacological effect through the above targets.

Hyperglycaemia is associated with poor prognosis in TBI patients. In addition to lowering the blood sugar, INS can also reduce the production of nitric oxide, reactive oxygen species, and TNFa, thereby reducing the infection rate of patients with $\mathrm{TBI}$ and improving the prognosis for the recovery of neurological functions ${ }^{[35-36]}$. IGF1 can promote the growth and differentiation of neurons and plays an important role in repair and nerve regeneration after central nervous system injury ${ }^{[37]}$. TNF is an important immunomodulatory and inflammatory factor involved in the inflammatory process that occurs after TBI

Page $11 / 35$ 
[38]. TP53 plays a regulatory role in controlling the cell cycle and initiating cell apoptosis and is also involved in DNA damage repair. ALB has the functions of protein transport and free radical scavenging and is an important extracellular antioxidant. IL6 plays an important role in inflammation, the immune response, and nerve trauma repair after TBI. After TBI, microglia promote nerve repair in an IL6-dependent manner ${ }^{[39]}$. SRC participates in cell development, proliferation, and apoptosis through multiple signalling pathways in the body. SRC activation aggravates the destruction of the blood-brain barrier and cerebral oedema after TBI ${ }^{[40]}$. STAT3 participates in the JAK-STAT signalling pathway after TBI and promotes the expression of IL6, TNF, and other inflammatory cytokines. VEGFA is a vascular endothelial cell growthstimulating factor that can promote the division of vascular endothelial cells, induce vascular remodelling, and participate in pathophysiological processes such as trauma and repair. VEGFA is an important cause of the destruction of the blood-brain barrier in TBI ${ }^{[41]}$. MMP9 is a zinc-dependent extracellular matrix-degrading enzyme involved in angiogenesis, inflammation, and other processes. After TBI, MMP9 can aggravate brain damage by destroying the blood-brain barrier and inducing neuronal apoptosis and the inflammatory response ${ }^{[42]}$.

Although the above approaches illuminate the "multitargets and multipathways" therapeutic actions of resveratrol in the treatment of $\mathrm{TBI}$, we need to be careful in using them to draw conclusions. Our approaches might not produce optimal results since the data of the study came from existing databases. The authenticity of databases may have a negatively influence on our results. Additionally, this study was performed in silico rather than under the actual condition, the results may not reflect all the real cell network characteristics in vivo. Therefore, further experiments studies are needed to confirm this hypothesis.

\section{Conclusions}

In summary, based on network pharmacology and molecular docking technology, we speculate that IL6, MMP9, INS, and SRC may be the key targets of resveratrol in the treatment of TBI. Resveratrol, through the regulation of signalling pathways such as AGE-RAGE, FoxO, insulin resistance, complement and coagulation cascades, HIF-1, calcium ion, and JAK-STAT, regulates oxidative stress, endocrine metabolism, inflammation, apoptosis, and the immune response to reduce brain damage. In addition, resveratrol can induce vascular remodelling, nerve regeneration, or wound repair to promote the recovery of nerve function after TBI. This study provides a theoretical basis for understanding the mechanism of resveratrol in the treatment of $\mathrm{TBI}$ and provides a valuable reference for further exploration.

\section{Abbreviations}

TCMSP

Traditional Chinese medicine systems pharmacology database and analysis platform; PPI:Protein-protein interaction; GO:Gene Ontology; KEGG:Kyoto Encyclopedia of Genes and Genomes; TBI:Traumatic brain injury; BP:Biological processes; MF:Molecular functions; CC:Cellular components; OB:Oral bioavailability; 
DL:Drug-likeness; BBB:Blood-brain barrier; INS:Insulin; IGF1:Human insulin-like growth factor 1; TNF:Tumor necrosis factor; TP53:Tumor suppressor P53; ALB:Plasma albumin; IL6:Interleukin 6; STAT3:Signal transducer and activator of transcription 3; VEGFA:Vascular endothelial growth factor A; MMP9:Matrix metalloproteinase 9; MAPK:Mitogen-activated protein kinase; PI3K:Phosphatidylinositol-3 kinase

\section{Declarations}

Ethics approval and consent to participate: The study was reviewed and approved by Ethics Committee of Hangzhou Hospital of Traditional Chinese Medicine.

Consent for publication: Not applicable.

Availability of data and materials: The datasets used and/or analysed during the current study are available from the corresponding author on reasonable request.

Competing Interests: All authors certify that they have no affiliations with or involvement in any organization or entity with any financial interest or non-financial interest in the subject matter or materials discussed in this manuscript.

Funding: This work was supported by grants from the Zhejiang Traditional Chinese Medicine Administration (CN) (NO. 2021ZA105). The funding body provided financial support and had no role in the design of the study, collection, analysis, interpretation of data, or writing the manuscript.

Authors ' contributions: Feifei Liu conceived and designed the study, and wrote the first draft of the manuscript. Lijun Han collected the targets and performed the network pharmacology analysis. Yujian Li contributed significantly to molecular docking verification and language polishing. Guangming Bao contributed significantly to edit pictures and revision of the manuscript. All authors were responsible for reviewing data. All authors read and approved the final manuscript.

Acknowledgements: Not applicable.

\section{References}

1. Jiang JY, Gao GY, Feng JF, et al. Traumatic brain injury in China. Lancet Neurol. 2019;18(3):286-95.

2. Gao G, Wu X, Feng J, et al. Clinical characteristics and outcomes in patients with traumatic brain injury in China: a prospective, multicenter, longitudinal, observational study. Lancet Neurol. 2020;19(8):670-7.

3. Fan Y, Li Y, Huang S, et al. Resveratrol-primed exosomes strongly promote the recovery of motor function in $\mathrm{SCl}$ rats by activating autophagy and inhibiting apoptosis via the PI3K signaling pathway. Neurosci Lett. 2020;736:135262. 
4. Ru J, Li P, Wang J, et al. TCMSP: a database of systems pharmacology for drug discovery from herbal medicines. J Cheminform. 2014;6:13.

5. Liu Z, Guo F, Wang Y, et al. BATMAN-TCM: a Bioinformatics Analysis Tool for Molecular mech ANism of Traditional Chinese Medicine. Sci Rep. 2016;6:21146.

6. Wishart DS, Feunang YD, Guo AC, et al. DrugBank 5.0: a major update to the DrugBank database for 2018. Nucleic Acids Res. 2018;46(D1):D1074-82.

7. Nickel J, Gohlke B-O, Erehman J, et al. SuperPred: update on drug classification and target predic tion. Nucleic Acids Res. 2014;42:W26-31.

8. Wang X, Shen Y, Wang S, et al. PharmMapper 2017 update: a web server for potential drug target identification with a comprehensive target pharmacophore database. Nucleic Acids Res. 2017;45(W1):W356-60.

9. Daina A, Michielin O, Zoete V. SwissTargetPrediction: updated data and new features for efficient prediction of protein targets of small molecules. Nucleic Acids Res. 2019;47(W1):W357-64.

10. Wang Y, Zhang S, Li F, et al. Therapeutic target database 2020: enriched resource for facilitating research and early development of targeted therapeutics. Nucleic Acids Res. 2020;48(D1):D1031-41.

11. Davis AP, Grondin CJ, Johnson RJ, et al. The Comparative Toxicogenomics Database: update 2019. Nucleic Acids Res. 2019;47(D1):D948-54.

12. Stelzer G, Rosen N, Plaschkes I, et al. The GeneCards Suite: From Gene Data Mining to Disease Genome Sequence Analyses. Curr Protoc Bioinforma. 2016;54:1.30.1-1.30.33.

13. Amberger JS, Bocchini CA, Schiettecatte F, et al. OMIM.org: Online Mendelian Inheritance in Man $\left(\mathrm{OMIM}{ }^{\circledR}\right)$, an online catalog of human genes and genetic disorders. Nucleic Acids Res. 2015;43:D789-98.

14. Rappaport N, Twik M, Plaschkes I, et al. MalaCards: an amalgamated human disease compendium with diverse clinical and genetic annotation and structured search. Nucleic Acids Res. 2017;45(D1):D877-87.

15. Consortium UniProt. UniProt: a worldwide hub of protein knowledge. Nucleic Acids Res. 2019;47(D1):D506-15.

16. Szklarczyk D, Gable AL, Lyon D, et al. STRING v11: protein-protein association networks with increased coverage, supporting functional discovery in genome-wide experimental datasets. Nucleic Acids Res. 2019;47(D1):D607-13.

17. Zhou Y, Zhou B, Pache L, et al. Metascape provides a biologist-oriented resource for the analysis of systems-level datasets. Nat Commun. 2019;10:1523.

18. Sterling T, Irwin JJ. ZINC 15-Ligand Discovery for Everyone. J Chem Inf Model.

19. 2015.;55(11):2324-37.

20. Berman HM, Westbrook J, Feng Z, et al. The Protein Data Bank. Nucleic Acids Res. 2000;28(1):23542. 
21. Wang W, Wang S, Liu T, et al. Resveratrol: Multi-Targets Mechanism on Neurodegenerative Dis eases Based on Network Pharmacology. Front Pharmacol. 2020;11:694.

22. Shi Z, Qiu W, Xiao G, et al. Resveratrol Attenuates Cognitive Deficits of Traumatic Brain Injury by Activating p38 Signaling in the Brain. Med Sci Monit. 2018;24:1097-103.

23. Subedi L, Lee JH, Gaire BP, et al. Sulforaphane Inhibits MGO-AGE-Mediated Neuroinflammation by Suppressing NF-KB, MAPK, and AGE-RAGE Signaling Pathways in Microglial Cells. Antioxidants (Basel Switzerland). 2020;9(9):E792.

24. Sun L, Zhao M, Liu M, et al. Suppression of FoxO3a attenuates neurobehavioral deficits after trau matic brain injury through inhibiting neuronal autophagy. Behav Brain Res. 2018;337:271-9.

25. Cao ZW, Li CW, Liu XC. Resveratrol improves the cerebral ischemia-reperfusion injury in rats through the SIRT1/Fox01 signal pathway. Acta Univ Med Anhui. 2019;54(5):719-23.

26. Franklin W, Krishnan B, Taglialatela G. Chronic synaptic insulin resistance after traumatic brain injury abolishes insulin protection from amyloid beta and tau oligomer-induced synaptic dysfunction. Sci Rep. 2019;9(1):8228.

27. Parry J, Hwang J, Stahel CF, et al. Soluble terminal complement activation fragment sC5b-9: a new serum biomarker for traumatic brain injury? Eur J Trauma Emerg Surg. 2020. http://www.ncbi.nlm.nih.gov/pubmed/32451568.

28. Ben Shimon M, Shavit-Stein E, Altman K, et al. Thrombin as Key Mediator of Seizure Develop ment Following Traumatic Brain Injury. Front Pharmacol. 2020;10:1532.

29. Esnault P, Mathais Q, D’Aranda E, et al. Ability of Fibrin Monomers to Predict Progressive Hem.

30. orrhagic Injury in Patients with Severe Traumatic Brain Injury. Neurocrit Care. 2020;33(1):182-95.

31. Fletcher-Sandersjöö A, Maegele M, Bellander B-M. Does Complement-Mediated Hemostatic Dis turbance Occur in Traumatic Brain Injury? A Literature Review and Observational Study Protocol. Int J Mol Sci. 2020;21(5):1596.

32. Wang K, Jing Y, Xu C, et al. HIF-1 a and VEGF Are Involved in Deferoxamine-Ameliorated Trau.

33. matic Brain Injury. J Surg Res. 2020;246:419-26.

34. Bae Y-H, Joo H, Bae J, et al. Brain injury induces HIF-1 a-dependent transcriptional activation of.

35. LRRK2 that exacerbates brain damage. Cell Death Dis. 2018;9(11):1125.

36. Shin JA, Oh S, Ahn J-H, et al. Estrogen receptor-mediated resveratrol actions on blood-brain bar rier of ovariectomized mice. Neurobiol Aging. 2015;36(2):993-1006.

37. Guo YX, Du RS. Progress of researches on mechanism of acupuncture for traumatic brain injury. Zhen Ci Yan Jiu. 2020;45(7):587-91.

38. Hixson KM, Cogswell M, Brooks-Kayal AR, et al. Evidence for a non-canonical JAK/STAT sig naling pathway in the synthesis of the brain's major ion channels and neurotransmitter receptors. BMC Genom. 2019;20(1):677.

39. Zhu C, Chen J, Pan J, et al. Therapeutic effect of intensive glycemic control therapy in patients with. 
40. traumatic brain injury. A systematic review and meta-analysis of randomized controlled trials.Med icine. (Baltimore). 2018;97(30):e11671.

41. Brabazon F, Bermudez $S$, Shaughness $M$, et al. The effects of insulin on the inflammatory activity of BV2 microglia. PLoS One. 2018;13(8):e0201878.

42. Littlejohn EL, Scott D, Saatman KE. Insulin-like growth factor-1 overexpression increases long-term survival of posttrauma-born hippocampal neurons while inhibiting ectopic migration following traumatic brain injury. Acta Neuropathol Commun. 2020;8(1):46.

43. Shao $X$, Yang $X$, Shen J, et al. TNF-a-induced p53 activation induces apoptosis in neurological in jury. J Cell Mol Med. 2020;24(12):6796-803.

44. Willis EF, MacDonald KPA, Nguyen QH, et al. Repopulating Microglia Promote Brain Repair in.

45. an IL-6-Dependent Manner. Cell. 2020;180(5):833-46.e16.

46. Liu D, Zhang X, Hu B, et al. Src Family Kinases in Brain Edema After Acute Brain Injury. Acta Neurochir Suppl. 2016;121. Available from: http://www.ncbi.nlm.nih.gov/pubmed/26463946.

47. Dyhrfort P, Shen Q, Clausen F, et al. Monitoring of Protein Biomarkers of Inflammation in Human Traumatic Brain Injury Using Microdialysis and Proximity Extension Assay Technology in Neurointensive Care. J Neurotrauma. 2019;36(20):2872-85.

48. Tang YL, Fang LJ, Zhong LY, et al. Hub genes and key pathways of traumatic brain injury: bioin formatics analysis and in vivo validation. Neural Regen Res. 2020;15(12):2262-9.

\section{Figures}




\section{Common Genes}

TBI

resveratrol

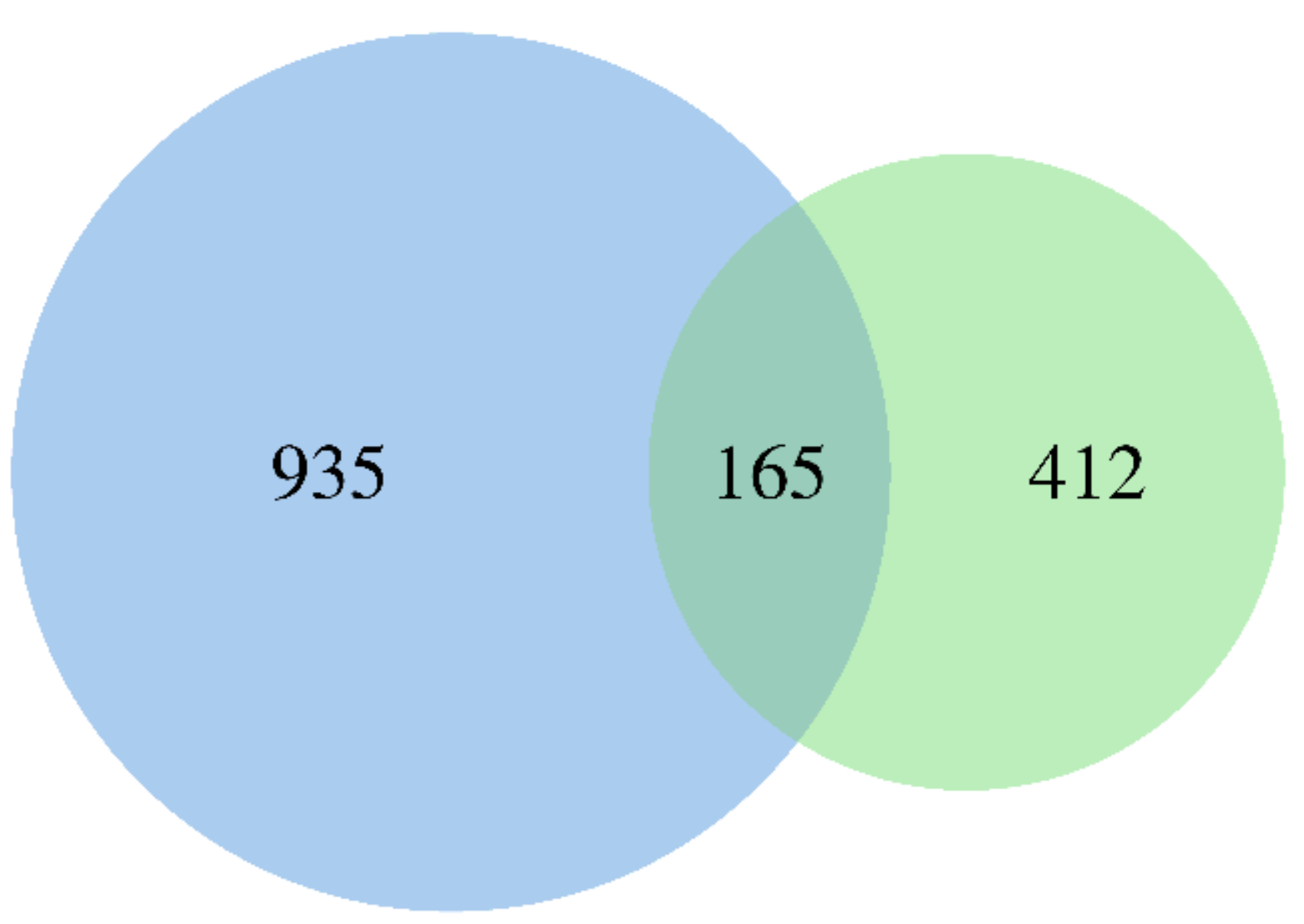

Figure 1

The overlapping targets of resveratrol and TBI 


\section{Common Genes}

TBI

resveratrol

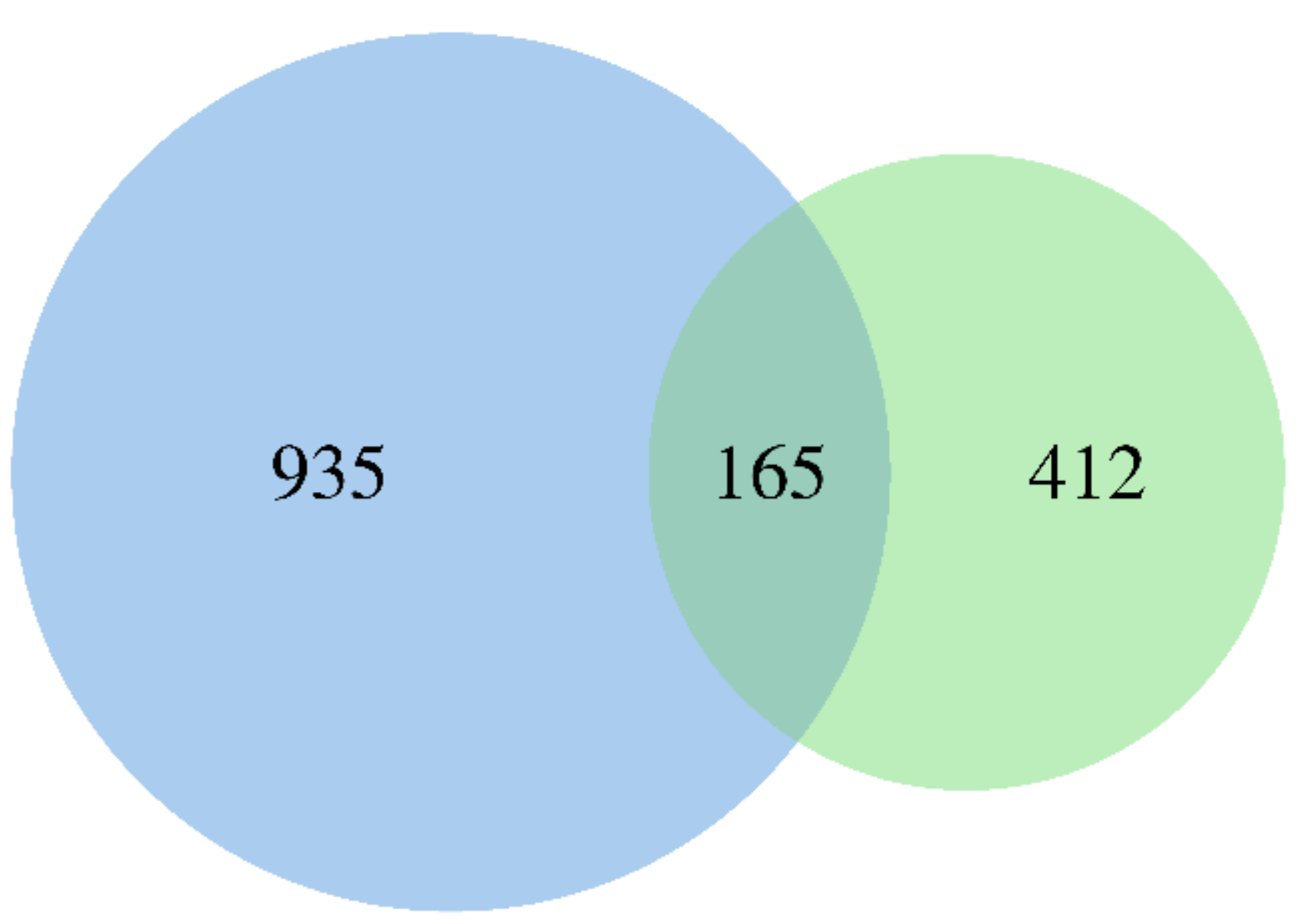

Figure 1

The overlapping targets of resveratrol and TBI 


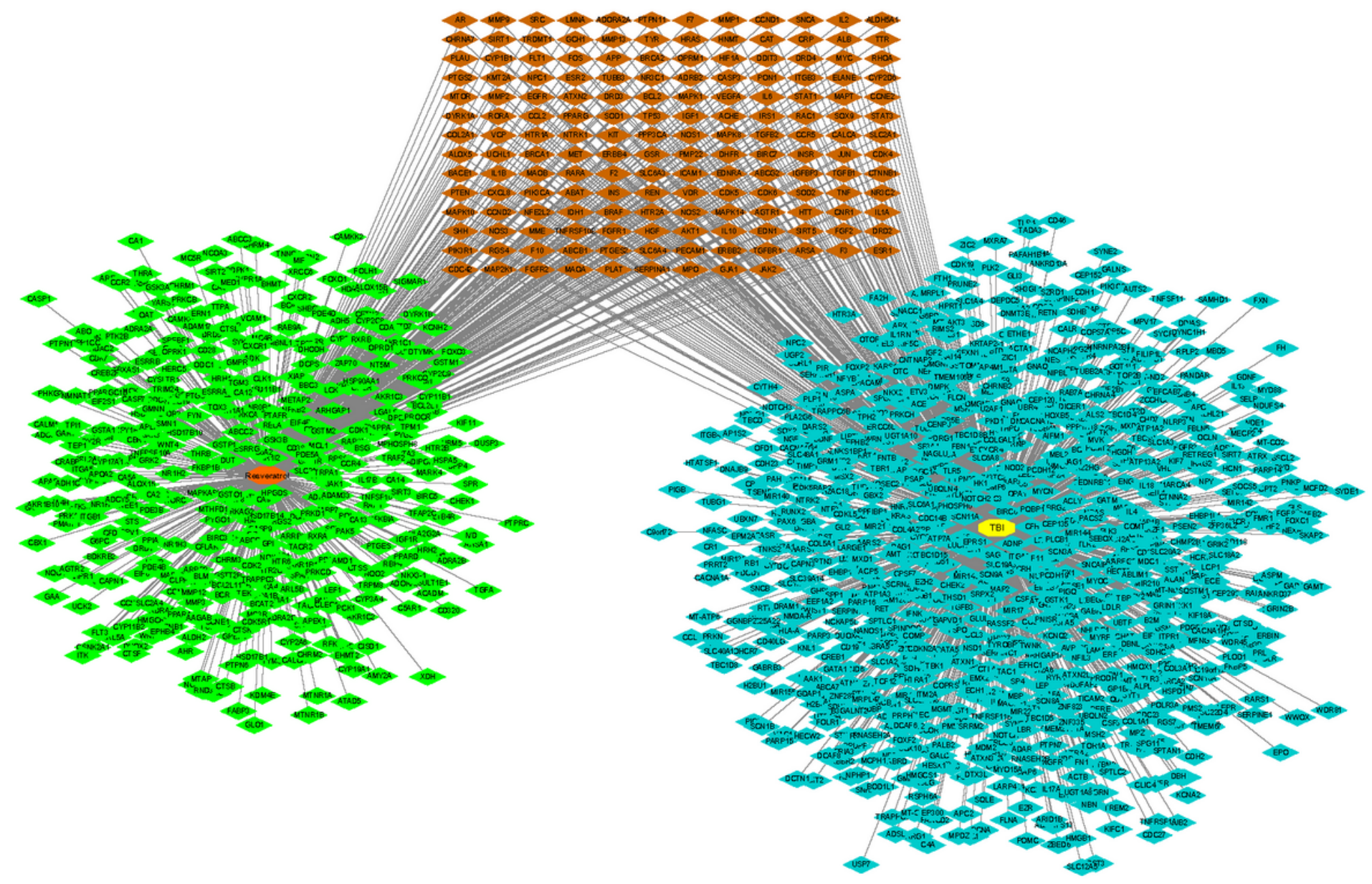

Figure 2

Drug-target-disease network of resveratrol in TBI treatment 


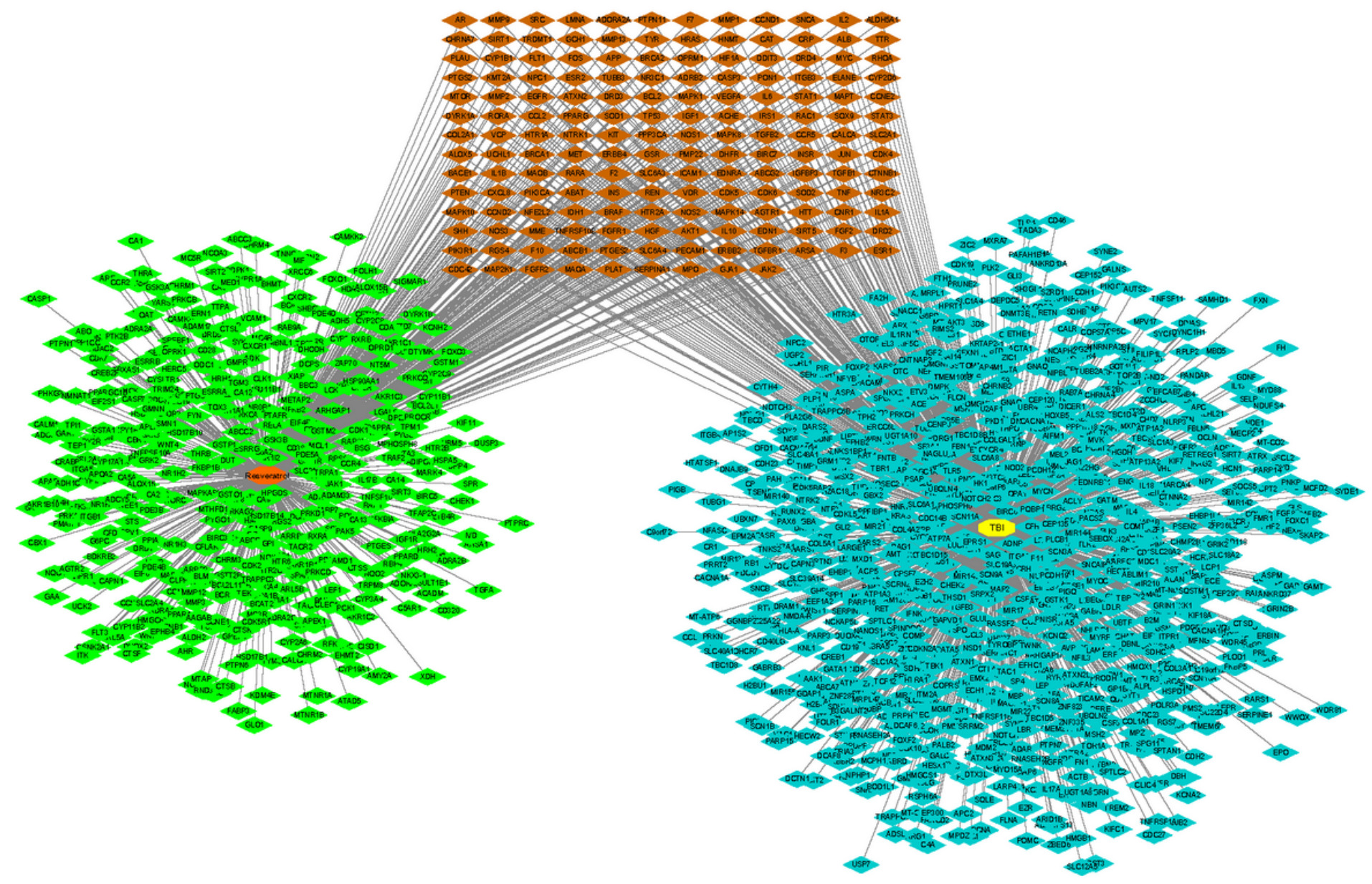

Figure 2

Drug-target-disease network of resveratrol in TBI treatment 


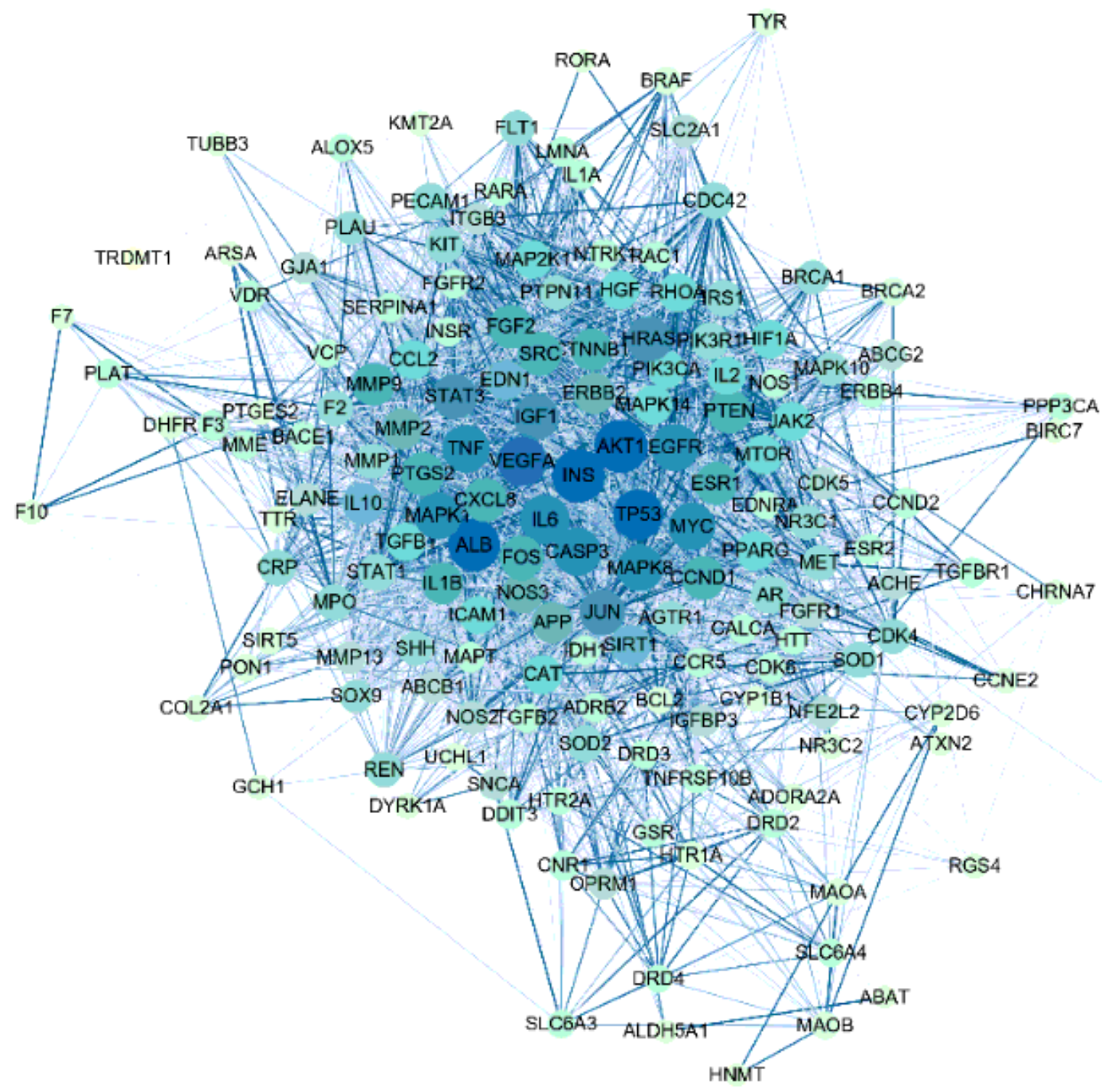

PMP22

NPC1

\section{Figure 3}

The PPI network of overlapping targets of resveratrol and TBI 


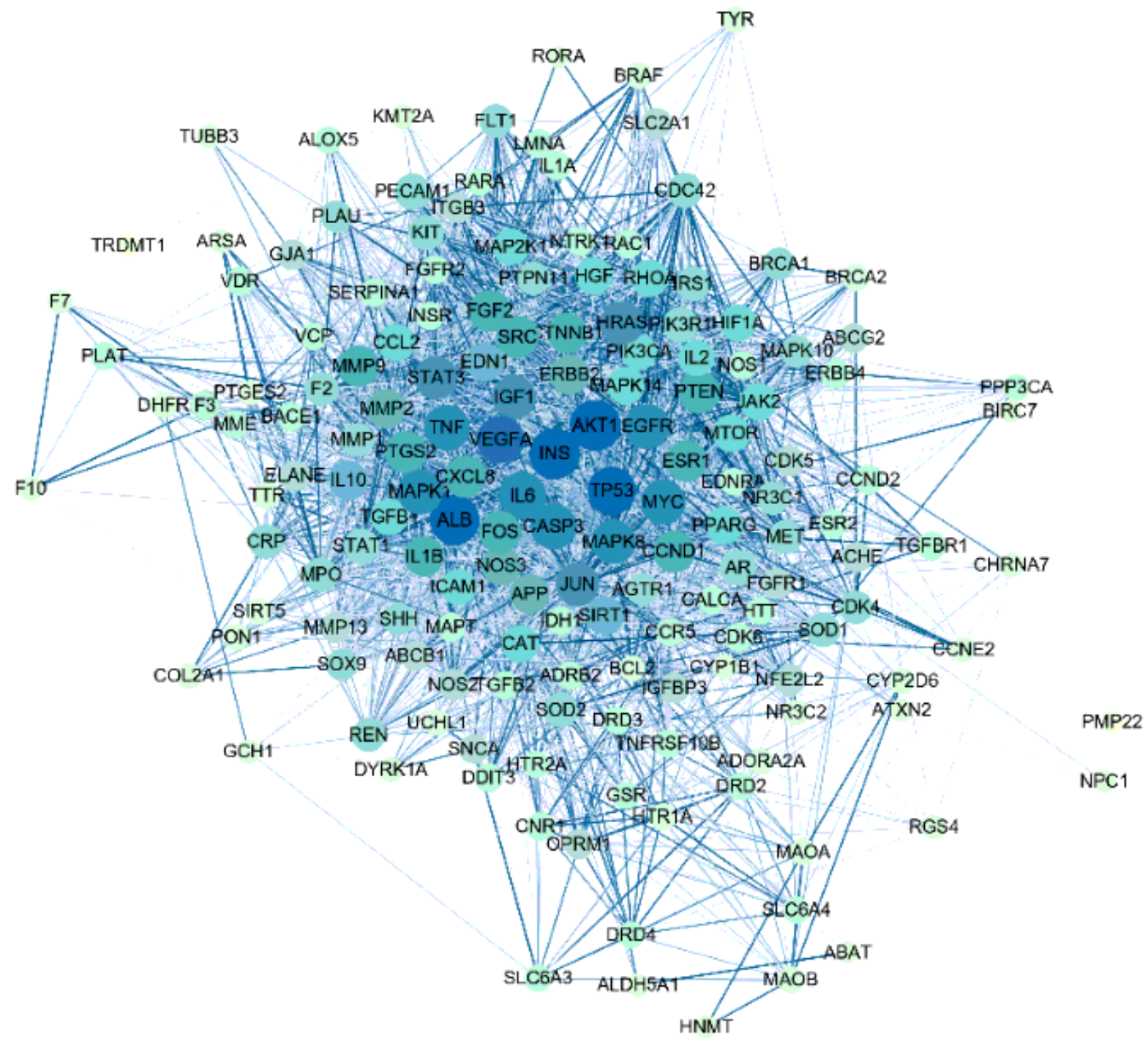

Figure 3

The PPI network of overlapping targets of resveratrol and TBI 


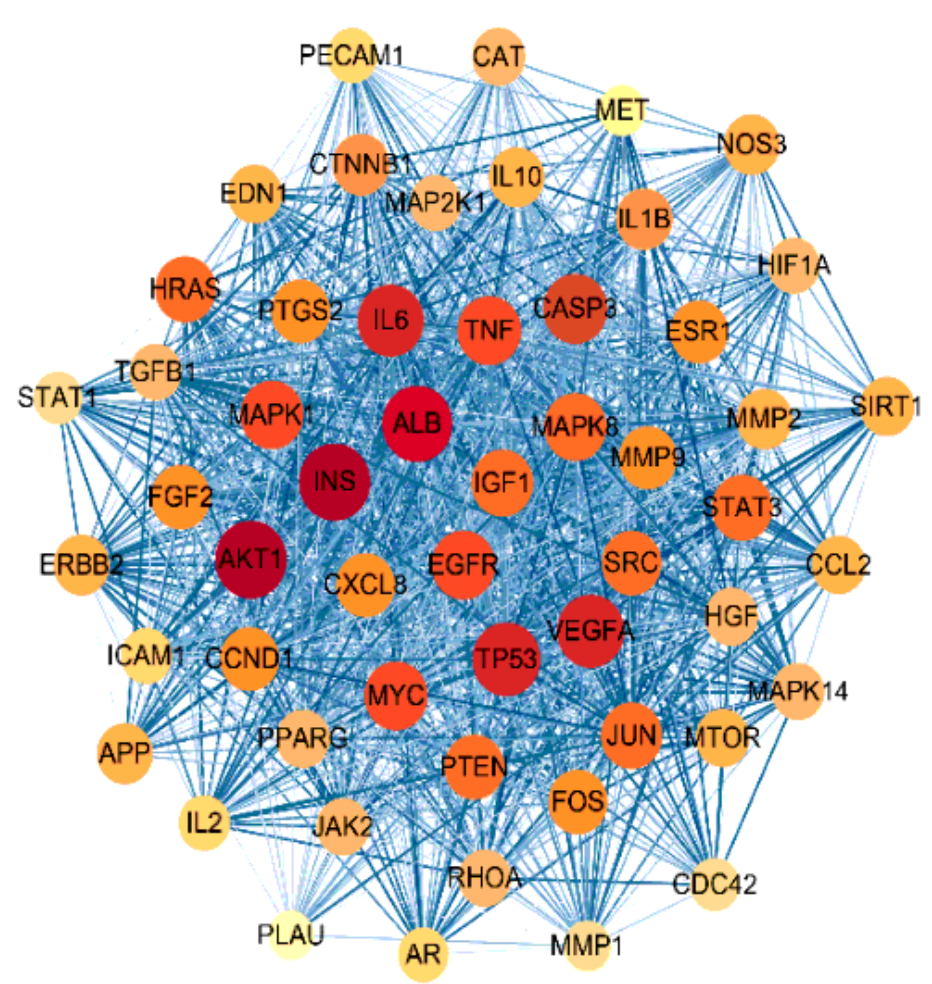

MCODE 1

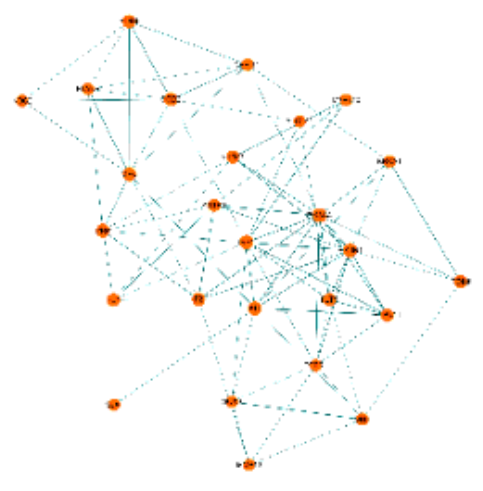

MCODE 2

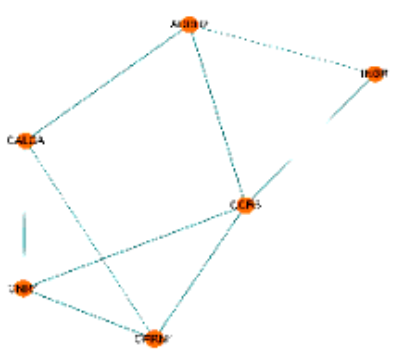

MCODE 4

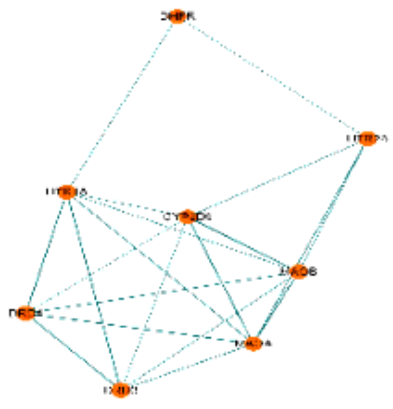

MCODE 3

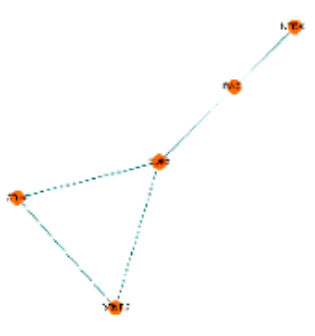

MCODE 5

\section{Figure 4}

Module analysis of overlapping targets

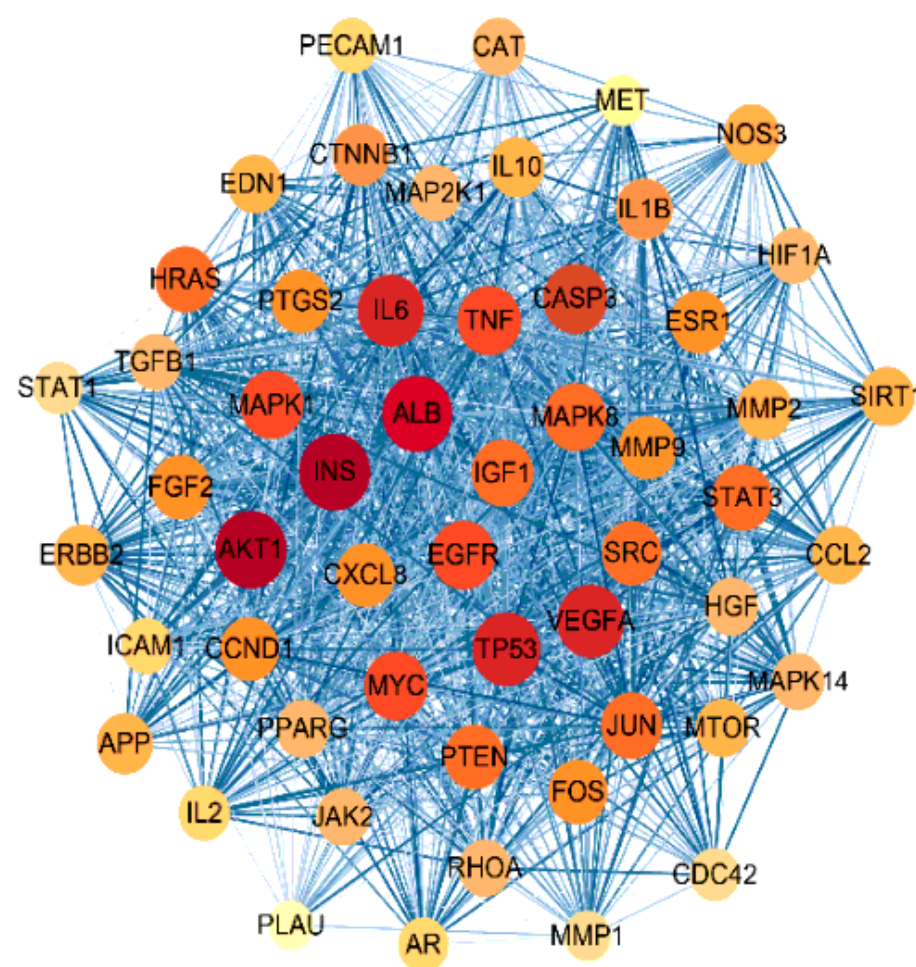

MCODE 1

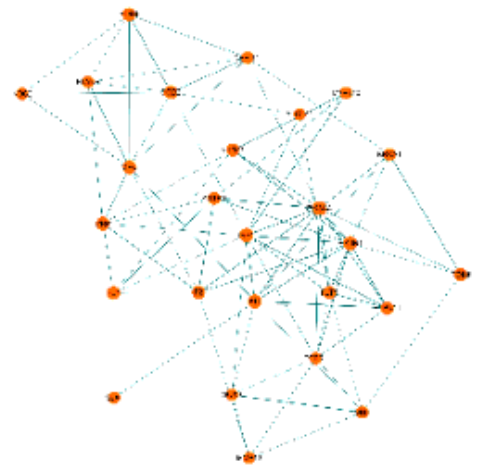

MCODE 2

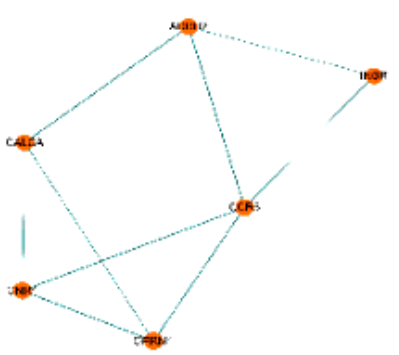

MCODE 4

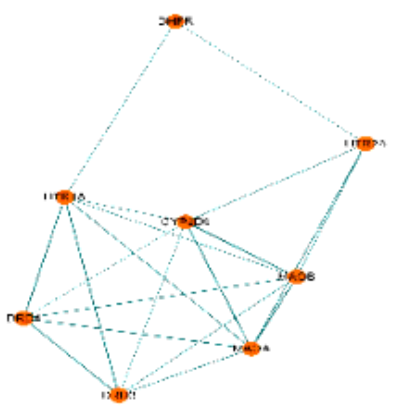

MCODE 3

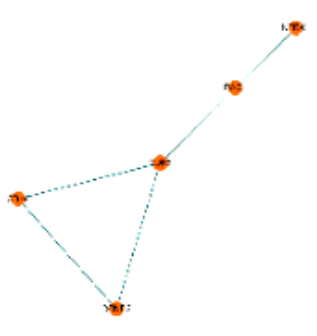

MCODE 5 
Figure 4

Module analysis of overlapping targets
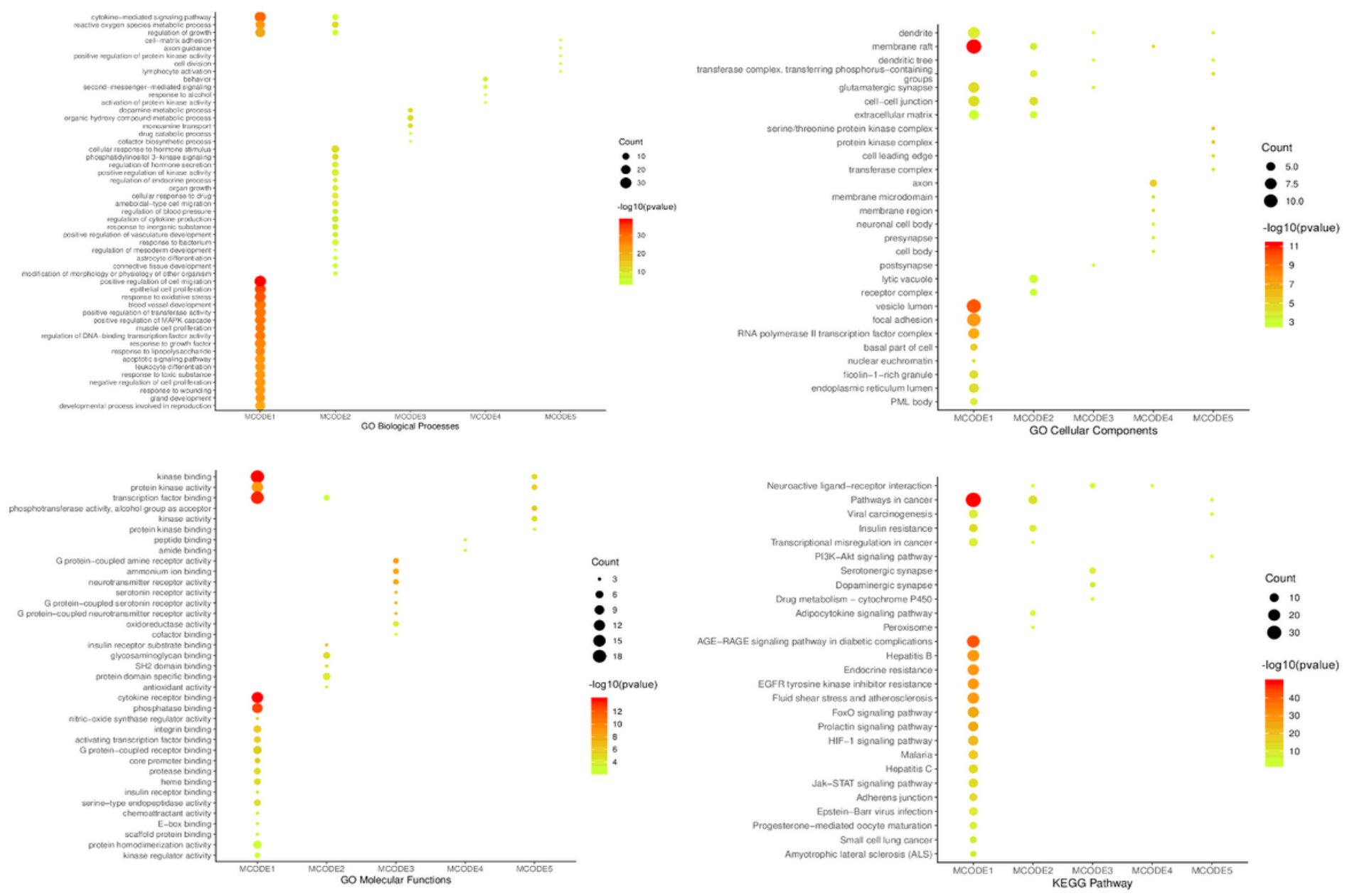

Figure 5

GO functional and KEGG pathway enrichment analysis of MCODE 1-5 

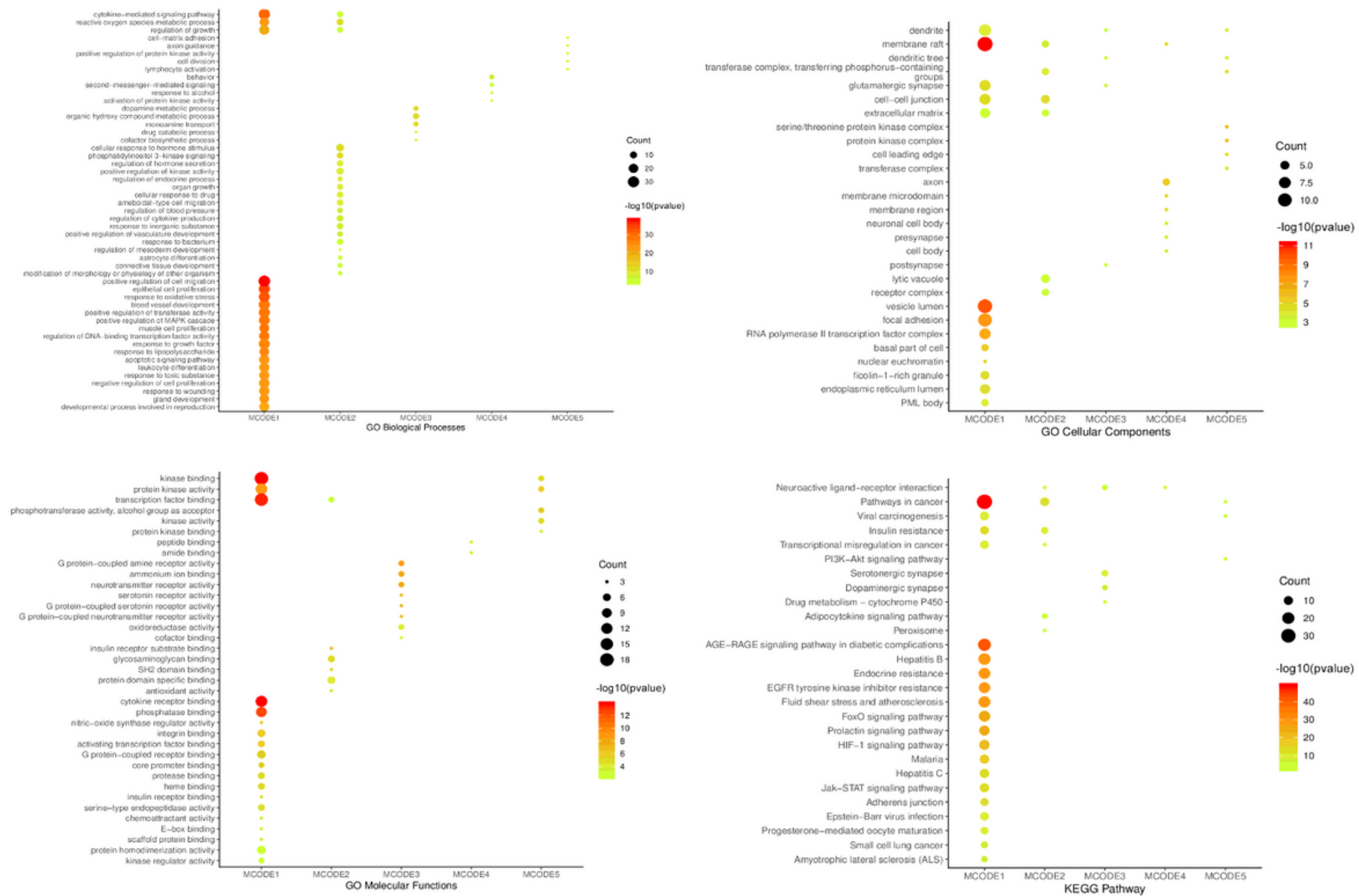

Figure 5

GO functional and KEGG pathway enrichment analysis of MCODE 1-5 


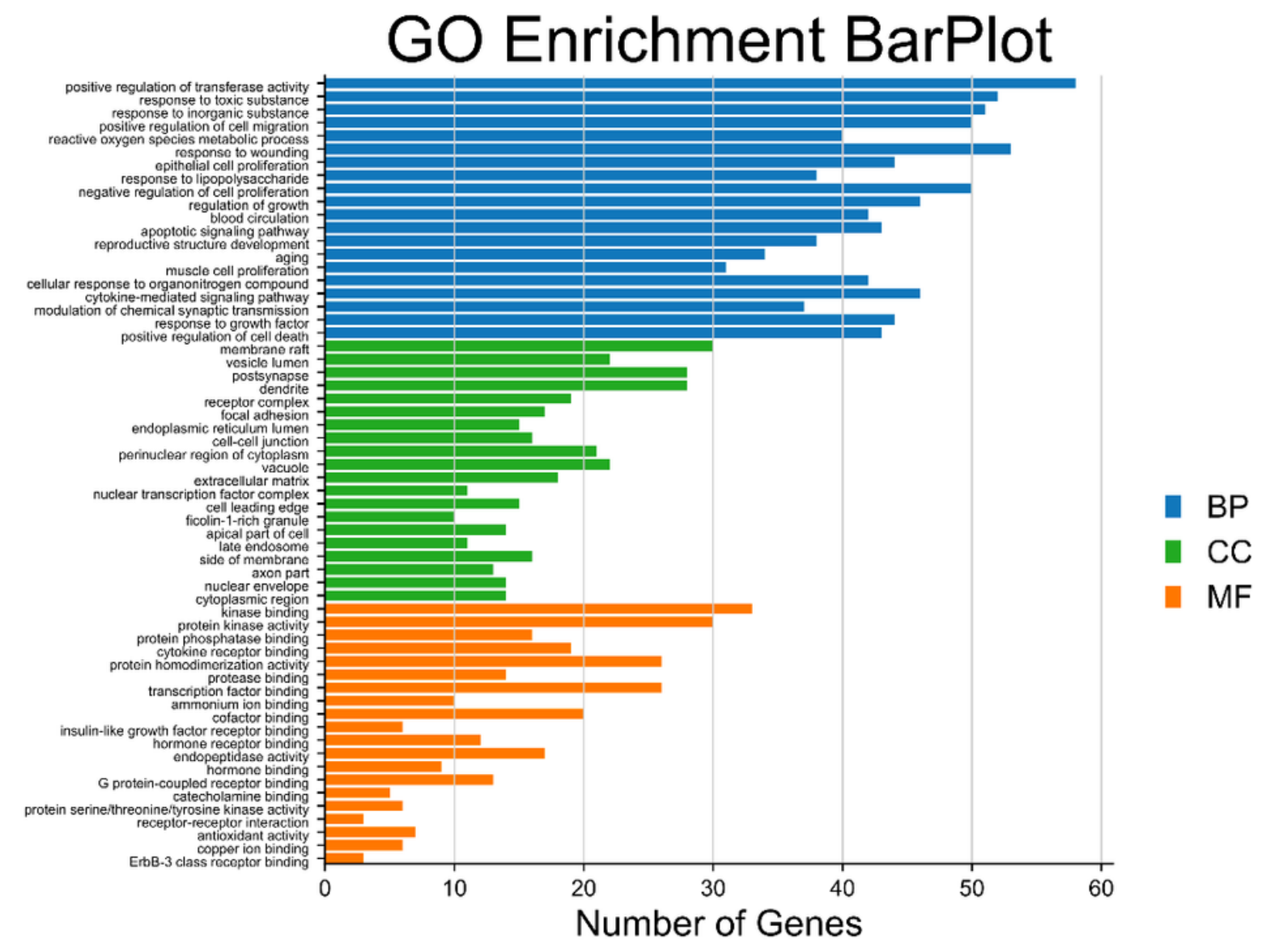

Figure 6

GO functional enrichment analysis of overlapping targets 


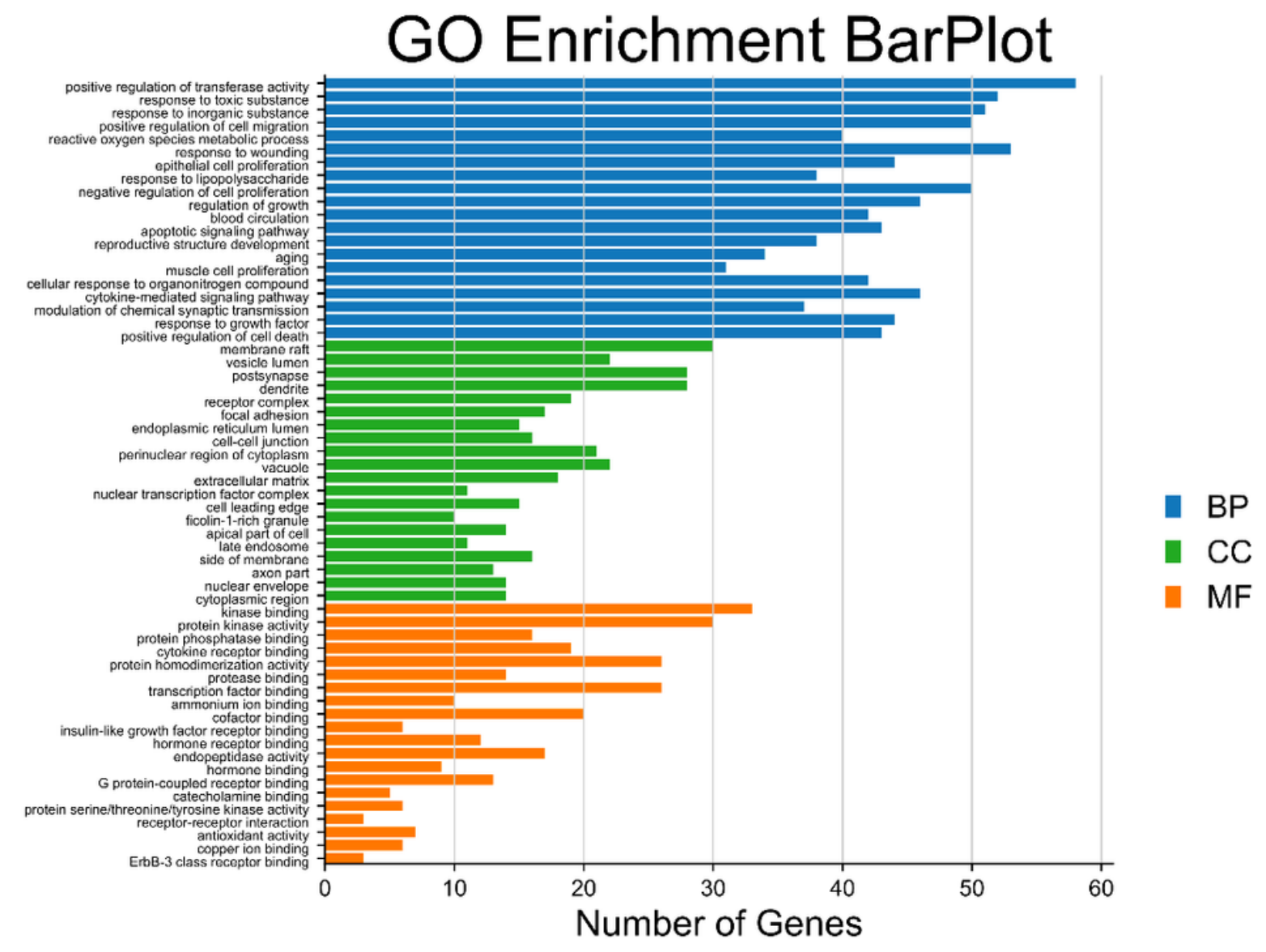

Figure 6

GO functional enrichment analysis of overlapping targets 


\section{TOP 20 of Pathway Enrichment}

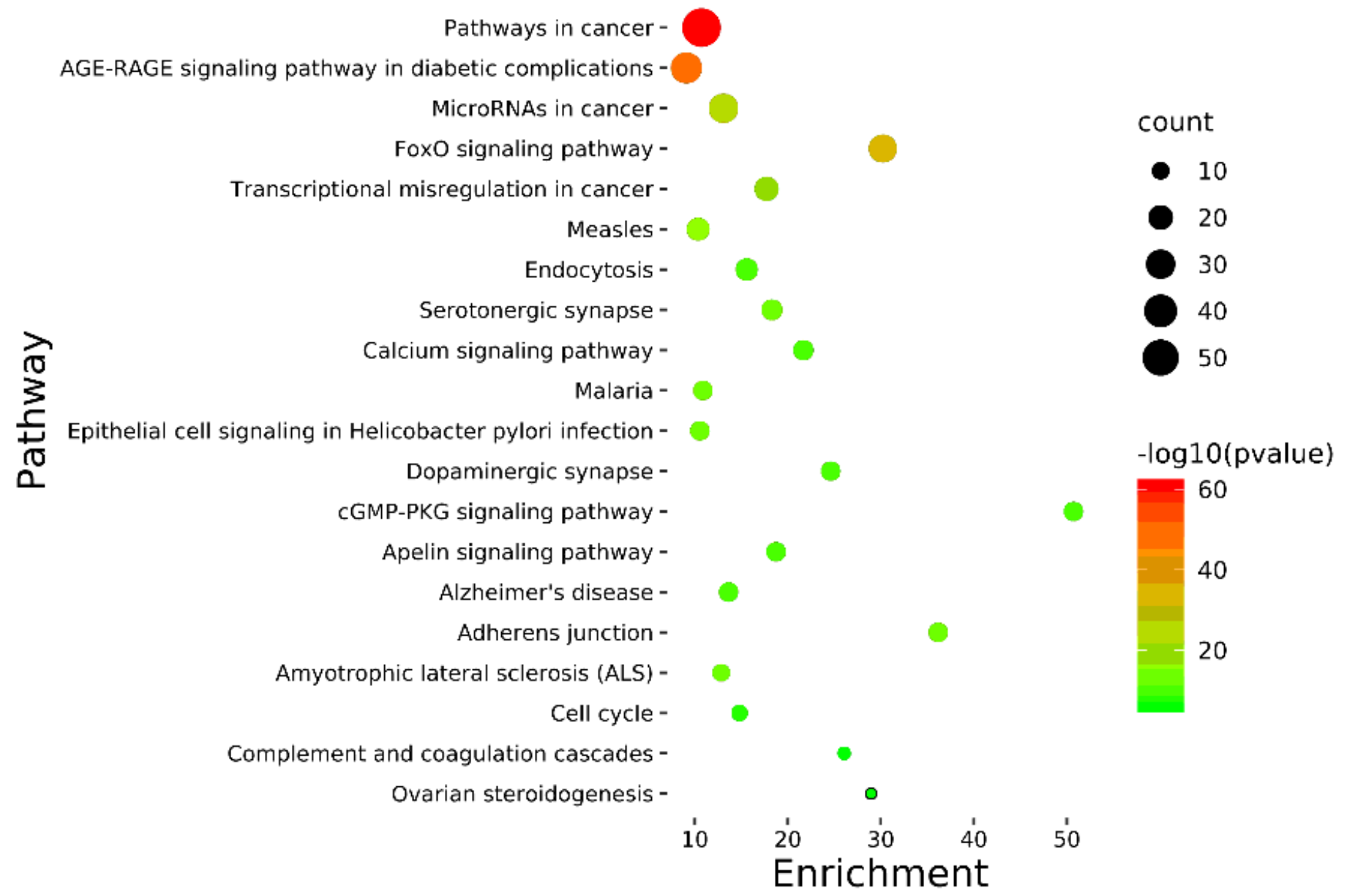

Figure 7

KEGG pathway enrichment analysis of overlapping targets 


\section{TOP 20 of Pathway Enrichment}

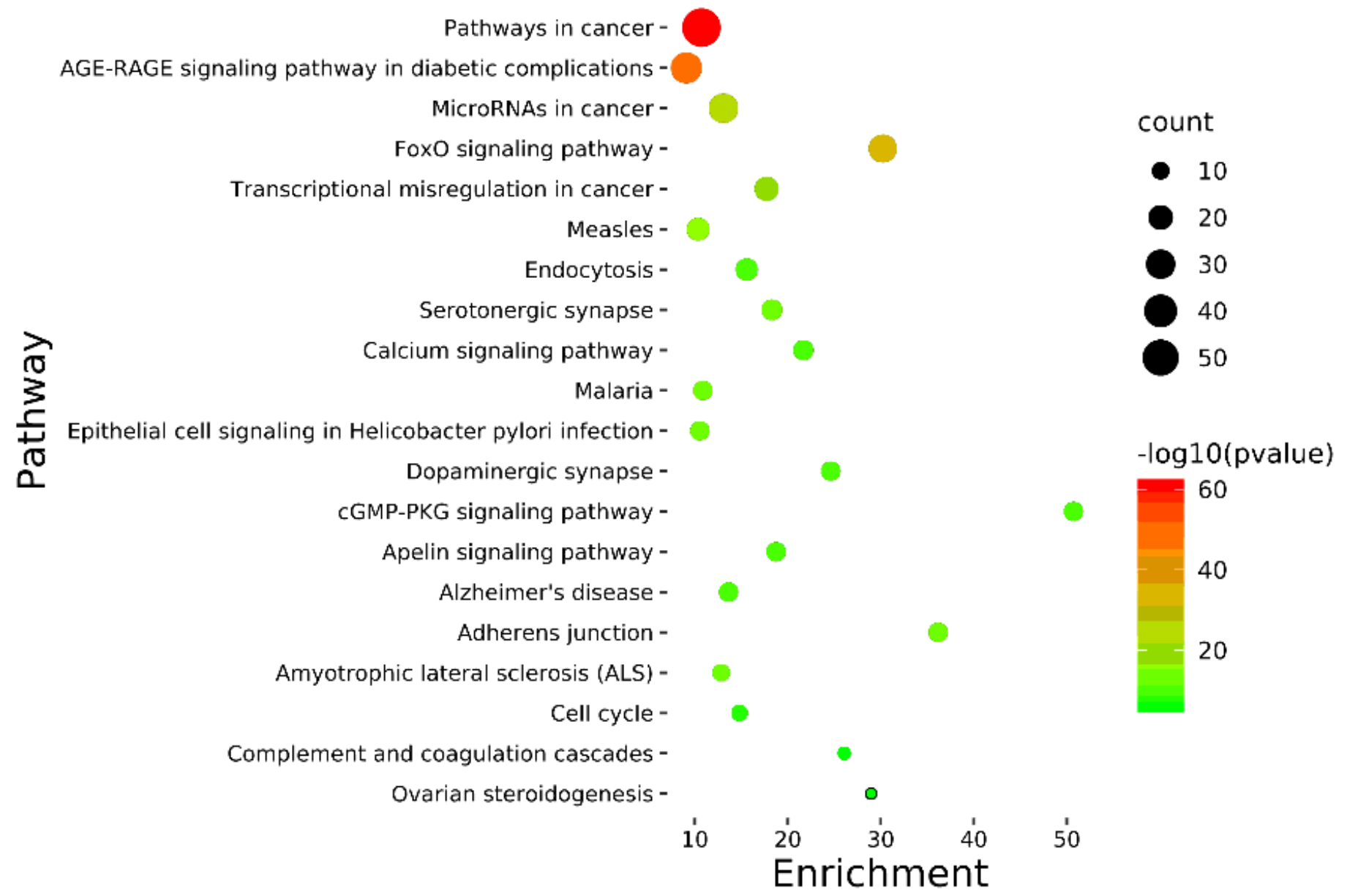

Figure 7

KEGG pathway enrichment analysis of overlapping targets 


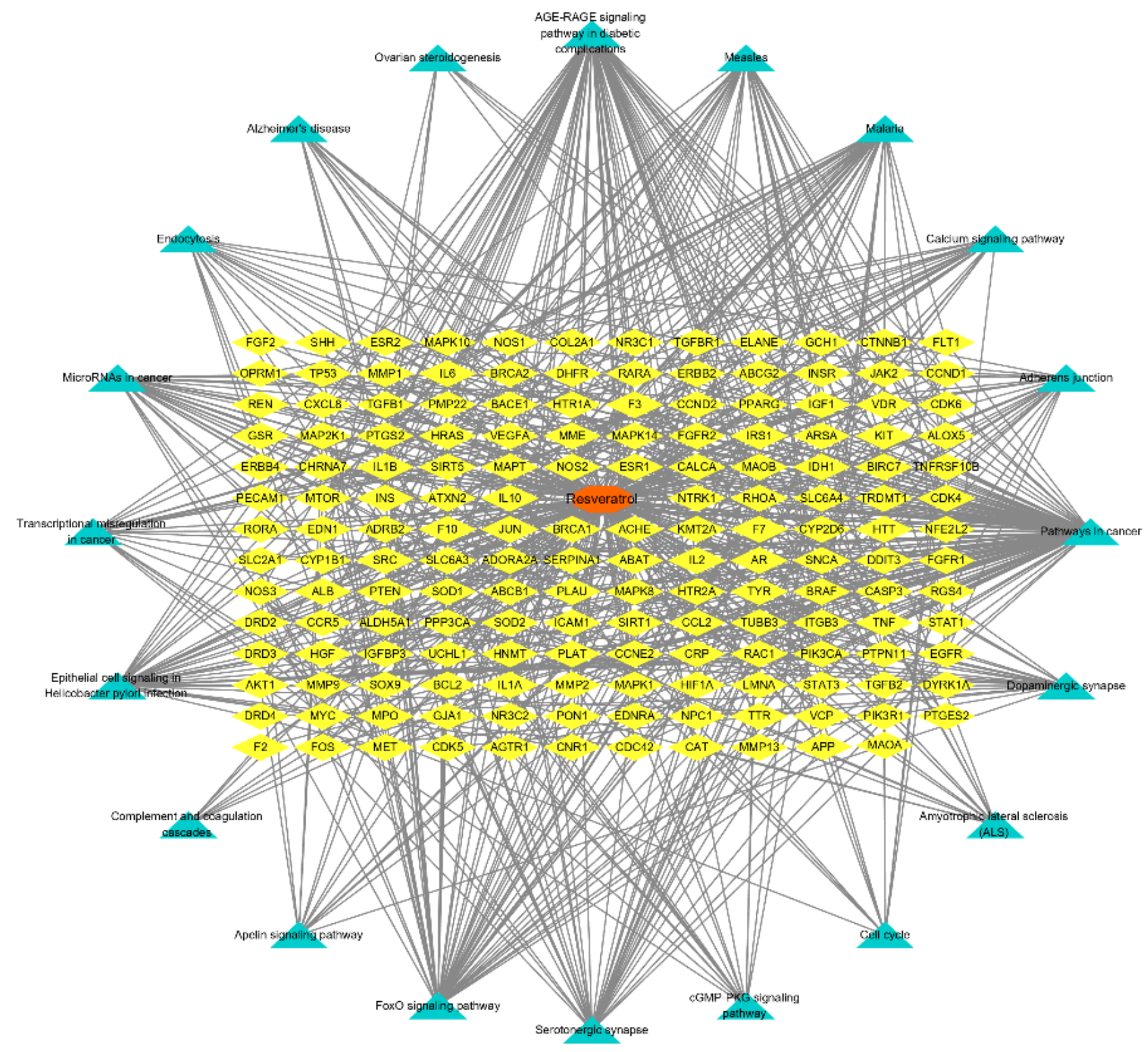

Figure 8

Drug-target-KEGG pathway network 


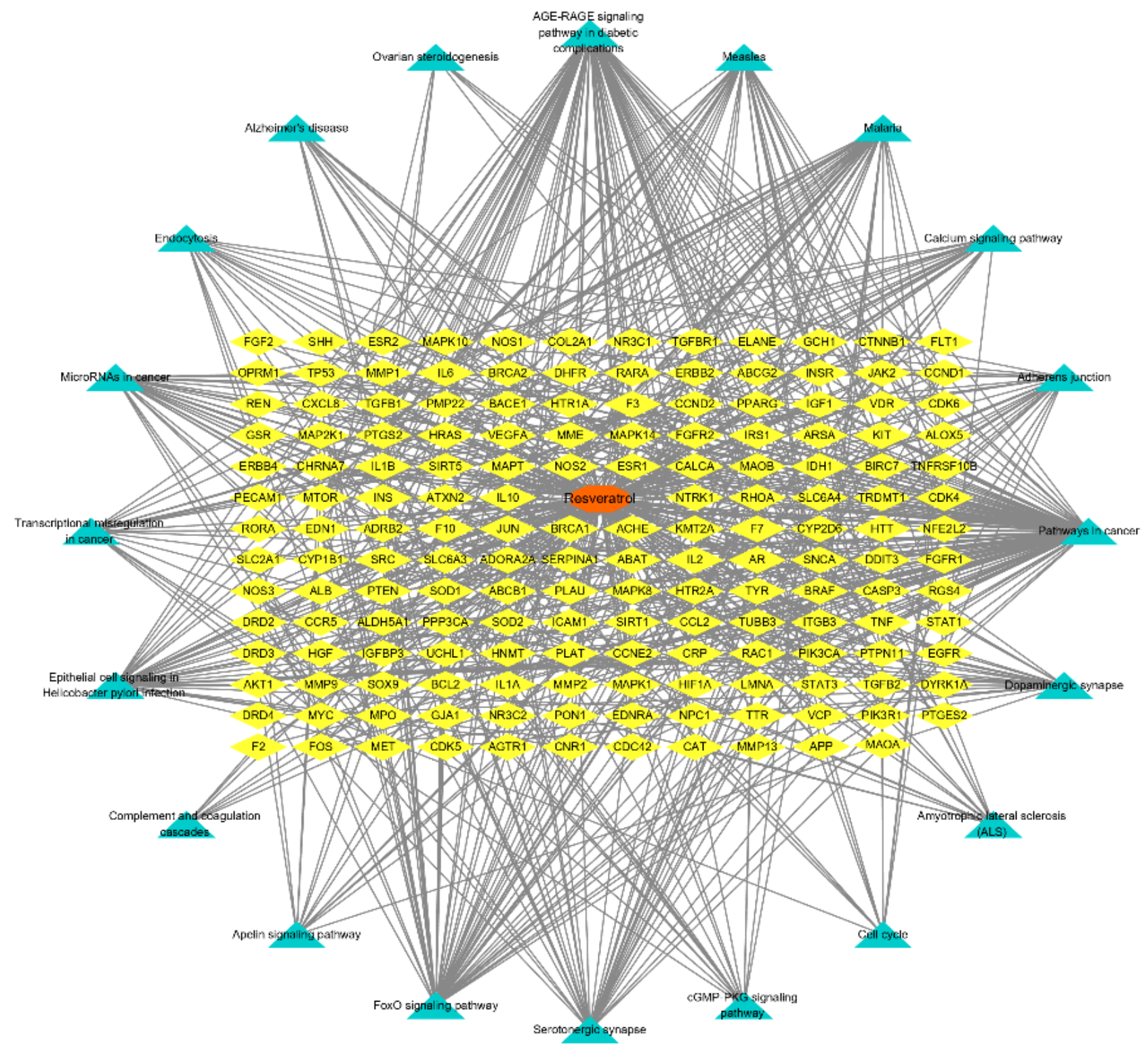

Figure 8

Drug-target-KEGG pathway network 


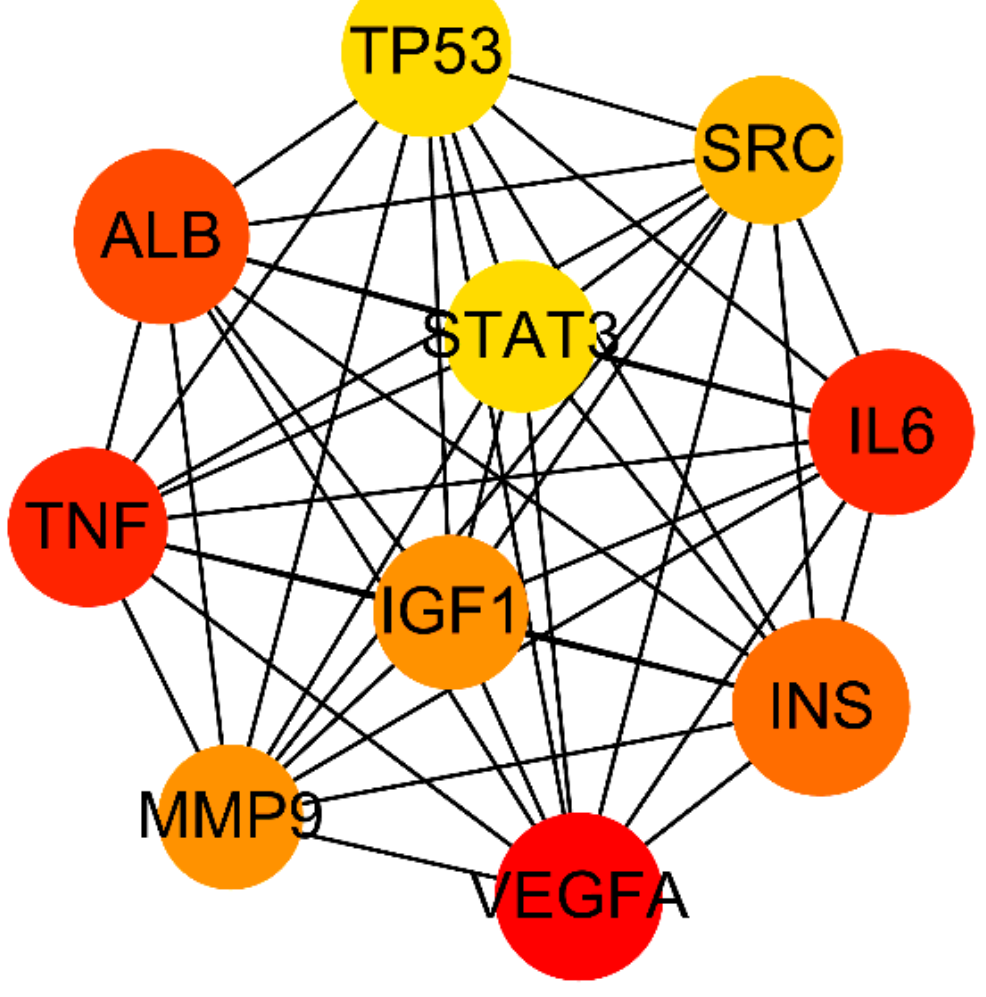

Figure 9

PPI network of the top 10 hub genes 


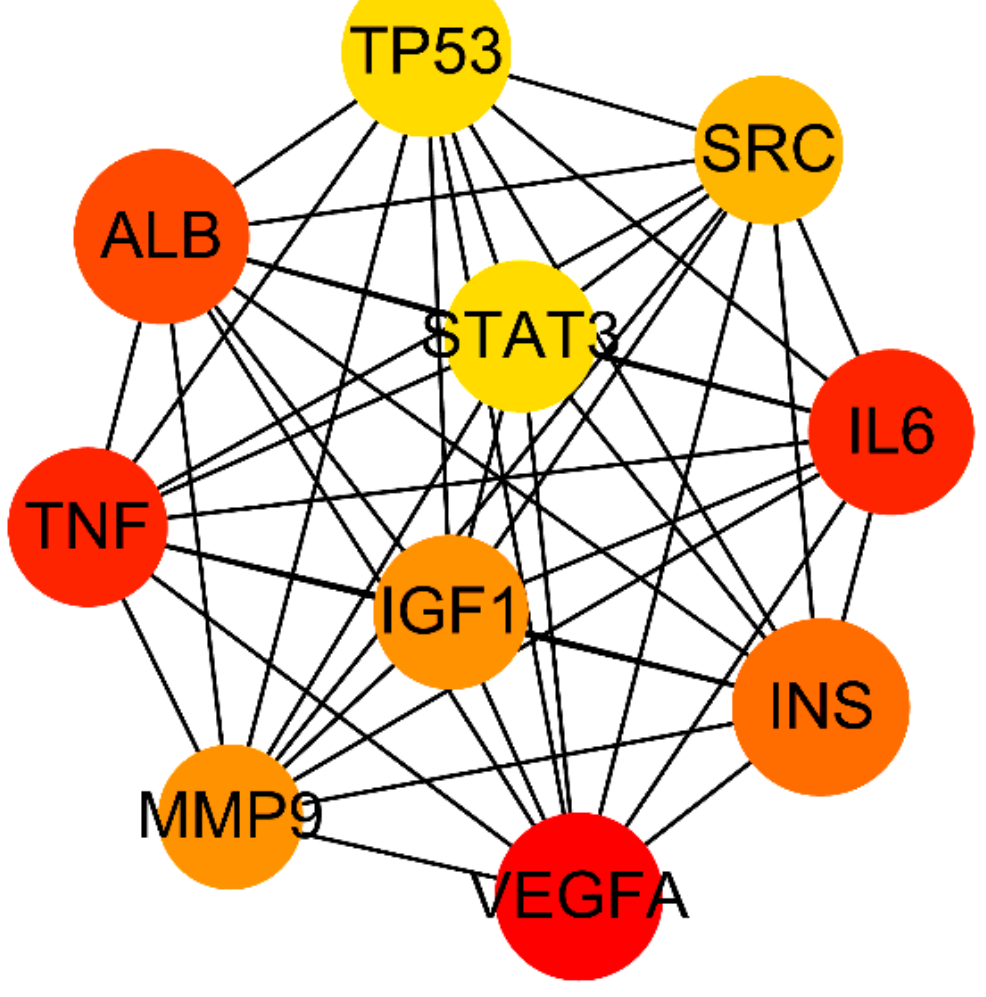

Figure 9

PPI network of the top 10 hub genes 


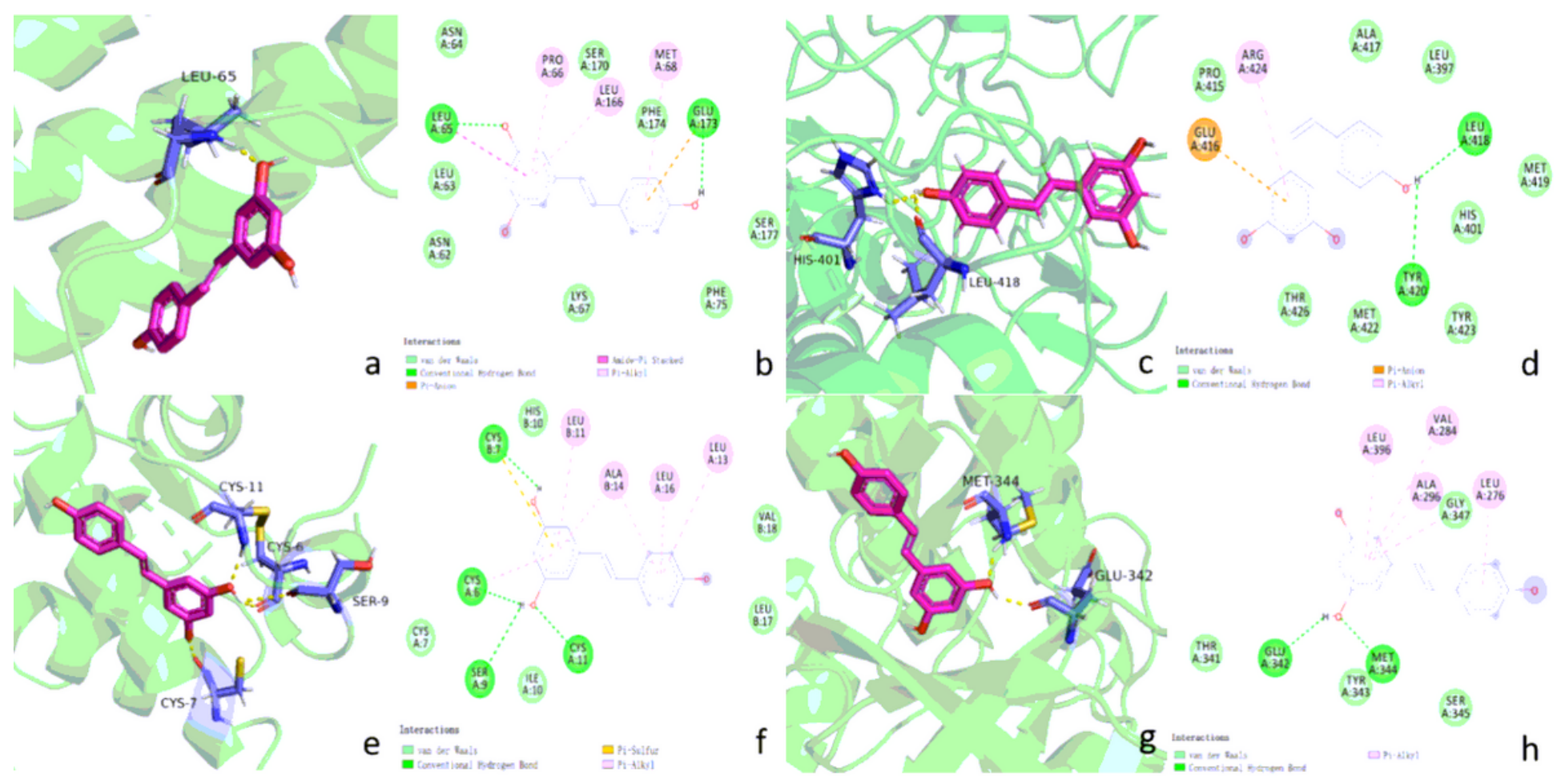

Figure 10

The 2D and 3D molecular docking patterns of resveratrol with IL6 (a, b), MMP9 (c, d), INS (e, f), and SRC $(\mathrm{g}, \mathrm{h})$

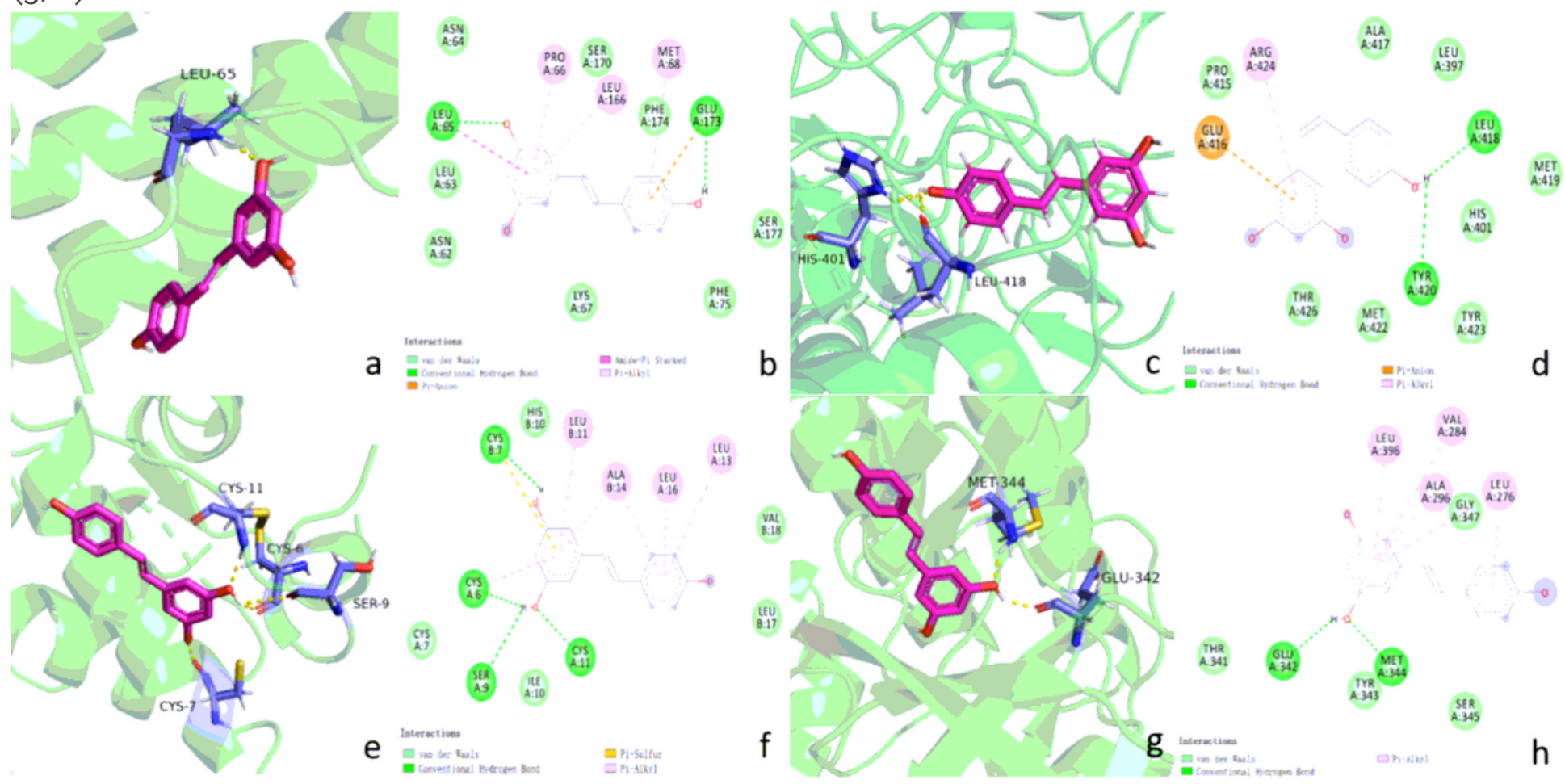

Figure 10 
The 2D and 3D molecular docking patterns of resveratrol with IL6 $(a, b), \operatorname{MMP9}(c, d)$, INS (e, f), and SRC $(\mathrm{g}, \mathrm{h})$ 